\title{
Chemoselective Synthesis of Novel Thiatriazolophanes
}

\author{
Madhukar S. Chande,* Kiran A. Puthamane, Pravin A. Barve, Rahul R. Khanwelkar and \\ Deepak S. Venkataraman
}

Department of Chemistry, The Institute of Science, 15, Madam Cama Road, Mumbai 400 032, India

Bis-[4-alquil/aril-5-tia-1,2,4-triazóis] foram preparados pela fusão de diácidos e tiosemicarbazidas, pela condensação de hidrazidas ácidas aromáticas com isotiocianato. A alquilação quimiosseletiva destes bis-[4-alquil/aril-5-tia-1,2,4-triazóis] com 1, $\omega$-dihaloalcanos na presença de hidróxido de potássio em metanol aquoso, proporcionou $\mathrm{N}$-alquil/aril tiatriazolofanos inéditos.

Bis-[4-alkyl/aryl-5-thia-1,2,4-triazoles] were prepared by fusion of diacids and thiosemicarbazides by condensation of aromatic acid hydrazides with isothiocyanate. Chemoselective alkylation of these bis-[4-alkyl/aryl-5-thia-1,2,4-triazoles] with 1, $\omega$-dihaloalkanes in presence of potassium hydroxide in aqueous methanol afforded novel $\mathrm{N}$-alkyl/aryl thiatriazolophanes.

Keywords: 4-alkyl/aryl-5-thia-1,2,4-triazoles, 1, $\omega$-dihaloalkanes, N-alkyl/aryl thiatriazolophanes, chemoselectivity

\section{Introduction}

Crown compounds have generated considerable interest during the last three decades because of their ability to form stable complexes with a variety of metal and organic cations and anions. ${ }^{1}$ They also have wide applications in phase transfer catalysis. ${ }^{2}$ In recent years various structural changes have been made to the basic "crown ether" structure in order to enhance the selective activity of the ligands. ${ }^{3}$ These changes involve the insertion of aromatic and/or heterocyclic ring systems into the macrocycles. Incorporation of heterocyclic subunit provides rigidity to the macrocycle and contributes in increasing the stability of complexes formed with both metals and organic cations. ${ }^{3}$ The development of crown compounds especially macrocyclic compounds containing heterocyclic subunit has gained importance due to their wide range of applications. Several reviews and monographs have been published which highlight their synthesis and application in synthetic organic chemistry as phase transfer catalysts and in analytical chemistry as ligand for complexation., ${ }^{2,4}$

*e-mail: mschande@yahoo.com
In recent years, attention has been increasingly paid to the synthesis of bisheterocyclic compounds, which exhibit various biological activities. ${ }^{5}$ including antibacterial, antifungicidal, tuberculostatic and plant growth regulative properties. Bisheterocyclic compounds displayed much better antibacterial activity than heterocyclic compounds. ${ }^{6}$ Various 1,2,4-triazoles are found to be associated with diverse pharmacological activities such as antiasthmatic, ${ }^{7}$ antiviral (ribavirin), ${ }^{8}$ antifungal (fluconazole), ${ }^{9}$ antimicrobial, ${ }^{10}$ antibacterial, ${ }^{11}$ insecticidal, ${ }^{11,12}$ amoebicidal,${ }^{13}$ hypnotic,${ }^{14}$ cytotoxic, ${ }^{15}$ and hypotensive ${ }^{16}$ activities. This moiety was also found in potent agonist and antagonist receptor ligands, ${ }^{17}$ in HIV-1 protease inhibitors ${ }^{18}$ and in thrombin inhibitors. ${ }^{19}$ Along with these significant pharmaceutical uses, 1,2,4-triazole derivatives are effectively used in polymers, dyestuff, photographic chemicals and agricultural chemicals. ${ }^{20}$

We have previously reported the chemoselective synthesis of novel oxadiazolophanes ${ }^{21}$ and $\mathrm{N}$-aminotriazolophanes. ${ }^{22}$ In continuation of this ongoing program in the synthesis of novel macrocyclic ligands ${ }^{21,22}$ their computational studies $^{21,22}$ and their application as phase transfer catalyst (PTC), ${ }^{22}$ we now report a facile chemoselective synthesis of novel N-alkyl/aryl thiatriazolophanes. 


\section{Results and Discussion}

The present work describes a versatile synthetic strategy for the chemoselective synthesis of novel N-alkyl/aryl thiatriazolophanes. Obtained heterophanes would be similar to lariat ether ${ }^{23}$ with $\mathrm{N}$-alkyl/aryl groups as side arm. The study of the stereochemistry of these compounds is of interest as the two N-alkyl/aryl groups would be either cis or trans to each other depending on the alkyl chain joining the two heterocyclic units and the thermodynamic stability of the molecule.

There are many methods described in the literature for the synthesis of $\mathrm{N}$-substituted-1,2,4-triazoles, ${ }^{20}$ but none of them concerns the synthesis of heterophanes. Bis N-alkyl/ aryl thiatriazoles 3a-c were prepared in good yield by direct fusion of adipic acid 1 with thiosemicarbazides $\mathbf{2 a - c}$, according to $\mathrm{Xu}$ method $^{24}$ (Scheme 1). This compound 3 containing thioamido groups has an amphoteric nature and can exist in tautomeric forms $3 \mathrm{~A}$ and $3 \mathrm{~B}$. On alkylation of 3 with 1, $\omega$-dihaloalkane, multiple products could form depending on the reactions conditions.

Reaction of compounds 3a-c with diiodoalkanes $\mathbf{4 a - f}$ in aq. methanol (80\%) in the presence of potassium hydroxide as a base gave only products $\mathbf{5 a - n}$ chemoselectively in good yield (Scheme 1, Table 1). The reaction was carried out in large excess of solvent to ensure intramolecular cyclisation (high dilution condition).

The elucidation of structures 5a-n was accomplished on the basis of their spectral data and elemental analysis (Table 1). For example, reaction of 3a with 1,2 diiodoethane $\mathbf{4 b}$ resulted in the formation of the desired
N-ethylthiatriazolophane $\mathbf{5} b$, confirmed on the basis of NMR spectra. ${ }^{1} \mathrm{H}$ NMR spectrum of the compound showed triplet at $\delta 4.10$ for $\mathrm{S}-\mathrm{CH}_{2}$ group of ethane chain. Absence of peak for $\mathrm{C}=\mathrm{S}$ in ${ }^{13} \mathrm{C}$ NMR spectrum confirmed the chemoselectivity of S-alkylation. It showed signal for $\mathrm{S}-\mathrm{CH}_{2}$ carbon at $32.1 \mathrm{ppm}$. From the above data compound $5 \mathrm{c}$ was identified as $1^{4}, 6^{4}$-diethyl-7,11dithia-1,6(3,5)-di-(1,2,4-triazola)cycloundecaphane. ${ }^{25}$ The other thiatriazolophanes 5a-n were similarly synthesized and characterized.

In order to improve the solubility of the thiatriazolophanes, it was thought to incorporate a phenyl nucleus into the structure, which would also help in complexation by PTC. Hence, the scope of the previous reaction was extended to the synthesis of benzotriazolophanes 9 (Scheme 2). Bistriazoles $\mathbf{8}$ was synthesized in high yield, by reacting isophthalic acid dihydrazide $\mathbf{6}$ with phenyl/o-tolyl isothiocyanates 7 .

The reaction of $\mathbf{8 a - b}$ with $\mathbf{4 a - g}$ in aqueous methanol (80\%) in the presence of potassium hydroxide as a base gave desired benzotriazolophanes 9a-l chemoselectively in moderate yield (Scheme 2, Table 1). Structures of the products were confirmed on the basis of spectral data and elemental analysis (Table 1). For example, reaction of $\mathbf{8 a}$ with 1,2-diiodoethane $\mathbf{4 b}$ resulted in the formation of desired benzotriazolophane 9a, confirmed on the basis of NMR spectra. ${ }^{1} \mathrm{H}$ NMR spectrum of the compound showed triplet at $\delta 3.46$ for $\mathrm{S}_{-} \mathrm{CH}_{2}$ group of ethane chain. Absence of peak for $\mathrm{C}=\mathrm{S}$ in ${ }^{13} \mathrm{C}$ NMR spectrum confirmed the chemoselectivity of S-alkylation. It showed signals for $\mathrm{S}-\mathrm{CH}_{2}$ carbons at $31.8 \mathrm{ppm}$.

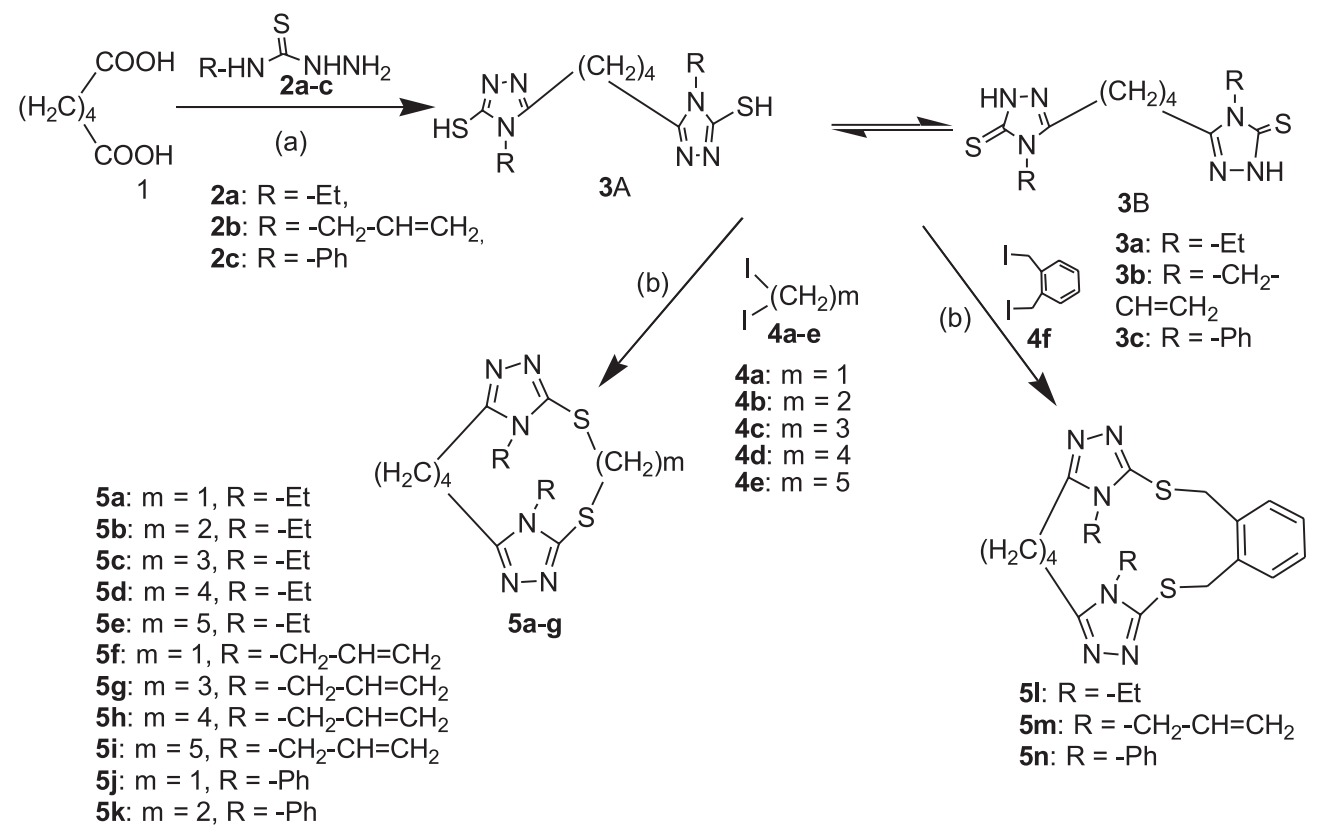

Reagents and conditions: (a) $140{ }^{\circ} \mathrm{C}$, fusion; (b) $\mathrm{KOH}$, aq. $\mathrm{MeOH}(80 \%), 80^{\circ} \mathrm{C}$.

Scheme 1. Synthesis of N-alkyl/aryl thiatriazolophanes $\mathbf{5}$. 


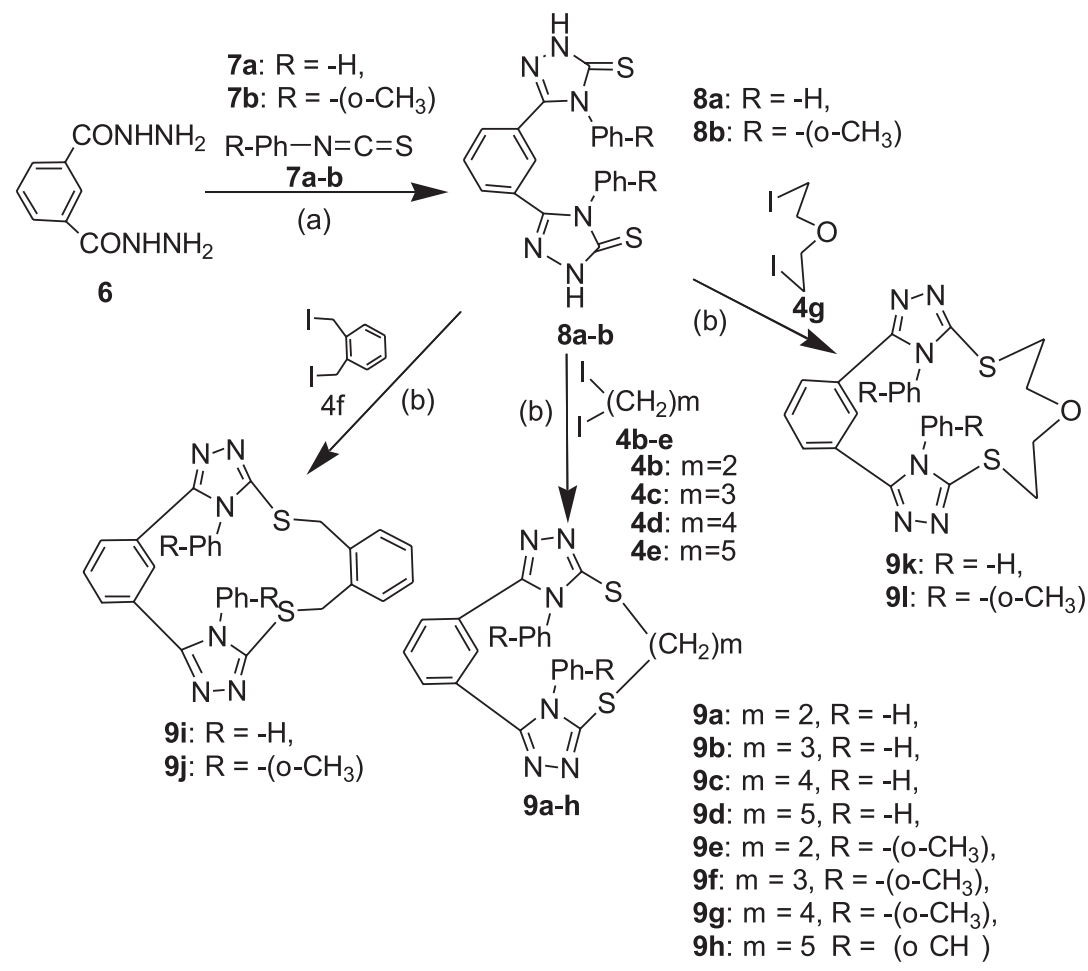

Reagents and conditions: (a) i) methanol, $75^{\circ} \mathrm{C}$, ii) $\mathrm{KOH}, 140{ }^{\circ} \mathrm{C}$, heating; (b) $\mathrm{KOH}$, aq. $\mathrm{MeOH}(80 \%), 80^{\circ} \mathrm{C}$.

Scheme 2. Synthesis of benzotriazolophanes 9.
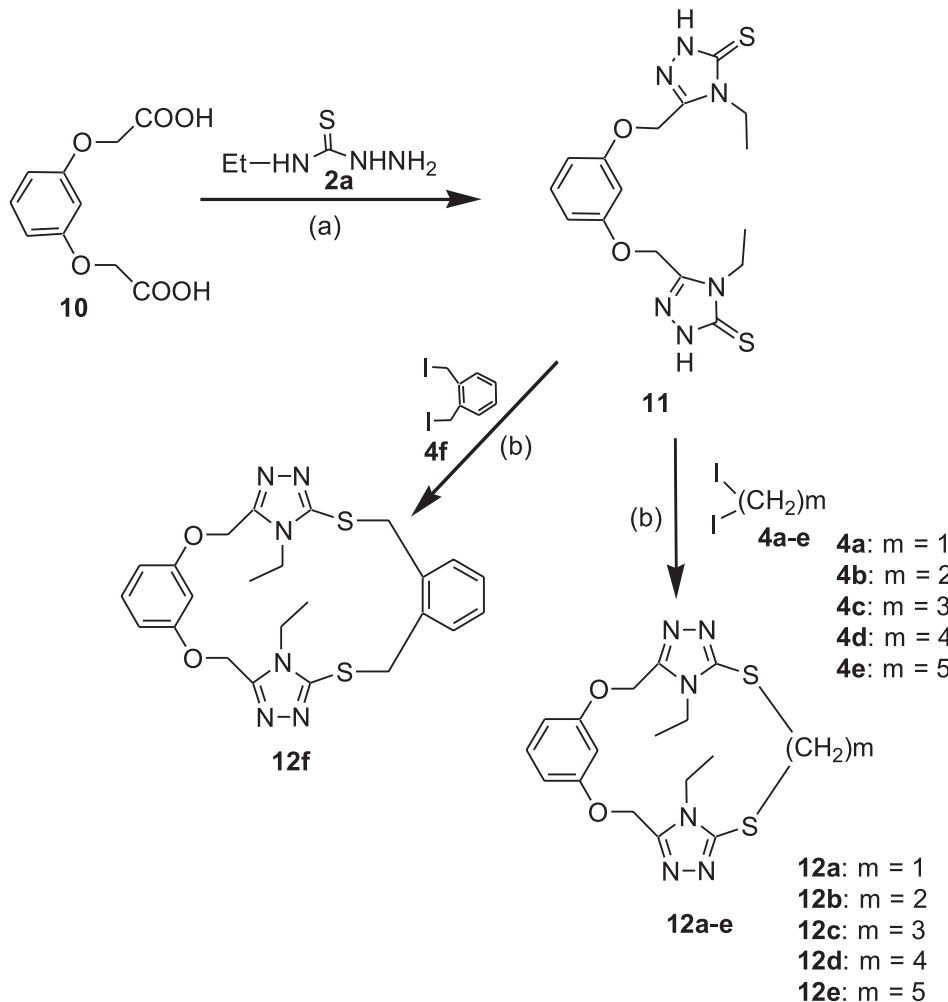

Reagents and conditions: (a) $140{ }^{\circ} \mathrm{C}$, fusion; (b) $\mathrm{KOH}$, aq. $\mathrm{MeOH}(80 \%), 80^{\circ} \mathrm{C}$.

Scheme 3. Synthesis of N-ethylthiatriazolophanes 12 
Table 1. Preparation and analytical data of compounds

\begin{tabular}{|c|c|c|c|c|c|c|c|}
\hline \multirow{2}{*}{ Compound } & \multirow{2}{*}{ Yield / \% } & \multirow{2}{*}{$\mathrm{mp} /{ }^{\circ} \mathrm{C}$} & \multirow{2}{*}{ Formulae } & \multicolumn{4}{|c|}{ Required (Found) / \% } \\
\hline & & & & $\mathrm{C}$ & $\mathrm{H}$ & $\mathrm{N}$ & $\mathrm{S}$ \\
\hline $\mathbf{3 a}$ & 80 & 250 & $\mathrm{C}_{12} \mathrm{H}_{20} \mathrm{~N}_{6} \mathrm{~S}_{2}$ & $46.10(46.00)$ & $6.41(6.26)$ & $26.90(26.80)$ & $20.50(20.37)$ \\
\hline $\mathbf{3 b}$ & 77 & 285 & $\mathrm{C}_{14} \mathrm{H}_{20} \mathrm{~N}_{6} \mathrm{~S}_{2}$ & $50.00(49.86)$ & $5.95(5.84)$ & $25.00(24.90)$ & $19.00(18.89)$ \\
\hline $3 c$ & 72 & $>300$ & $\mathrm{C}_{17} \mathrm{H}_{14} \mathrm{~N}_{6} \mathrm{~S}_{2}$ & $58.82(58.66)$ & $4.90(4.80)$ & $20.58(20.46)$ & $15.68(15.56)$ \\
\hline $5 \mathbf{a}$ & 48 & 272 & $\mathrm{C}_{13} \mathrm{H}_{20} \mathrm{~N}_{6} \mathrm{~S}_{2}$ & $48.12(47.99)$ & $6.21(6.07)$ & $25.90(25.78)$ & $19.76(19.68)$ \\
\hline $5 \mathbf{b}$ & 49 & 265 & $\mathrm{C}_{14} \mathrm{H}_{22} \mathrm{~N}_{6} \mathrm{~S}_{2}$ & $49.68(46.59)$ & $6.55(6.41)$ & $24.83(24.69)$ & $18.95(18.81)$ \\
\hline $5 c$ & 39 & Semisolid & $\mathrm{C}_{15} \mathrm{H}_{24} \mathrm{~N}_{6} \mathrm{~S}_{2}$ & $51.11(50.98)$ & $6.86(6.75)$ & $23.84(23.74)$ & $18.19(18.06)$ \\
\hline $5 d$ & 43 & 125 & $\mathrm{C}_{16} \mathrm{H}_{26} \mathrm{~N}_{6} \mathrm{~S}_{2}$ & $52.43(52.29)$ & $7.15(7.04)$ & $22.93(22.81)$ & $17.50(17.36)$ \\
\hline $5 e$ & 41 & 87 & $\mathrm{C}_{17} \mathrm{H}_{28} \mathrm{~N}_{6} \mathrm{~S}_{2}$ & $53.65(53.52)$ & $7.42(7.31)$ & $22.08(22.00)$ & $16.85(16.75)$ \\
\hline $5 f$ & 35 & 185 & $\mathrm{C}_{20} \mathrm{H}_{26} \mathrm{~N}_{6} \mathrm{~S}_{2}$ & $51.70(51.58)$ & $5.78(5.68)$ & $24.12(24.02)$ & $18.40(18.25)$ \\
\hline $5 \mathrm{~g}$ & 45 & 240 & $\mathrm{C}_{15} \mathrm{H}_{20} \mathrm{~N}_{6} \mathrm{~S}_{2}$ & $54.23(54.08)$ & $6.42(6.31)$ & $22.32(22.23)$ & $17.03(16.91)$ \\
\hline $5 \mathbf{h}$ & 37 & Semisolid & $\mathrm{C}_{17} \mathrm{H}_{24} \mathrm{~N}_{6} \mathrm{~S}_{2}$ & $55.35(55.21)$ & $6.71(6.57)$ & $21.52(21.41)$ & $16.42(16.29)$ \\
\hline $5 \mathbf{i}$ & 38 & Semisolid & $\mathrm{C}_{18} \mathrm{H}_{26} \mathrm{~N}_{6} \mathrm{~S}_{2}$ & $56.40(56.31)$ & $6.98(6.86)$ & 20.77 (20.67) & $15.85(15.71)$ \\
\hline $\mathbf{5 j}$ & 35 & Semisolid & $\mathrm{C}_{19} \mathrm{H}_{28} \mathrm{~N}_{6} \mathrm{~S}_{2}$ & $59.97(59.91)$ & 4.79 (4.68) & $19.98(19.92)$ & $15.25(15.16)$ \\
\hline $5 \mathbf{k}$ & 43 & 105 & $\mathrm{C}_{22} \mathrm{H}_{26} \mathrm{~N}_{6} \mathrm{~S}_{2}$ & $60.80(60.71)$ & $5.10(4.99)$ & 19.34 (19.29) & $14.76(14.66)$ \\
\hline 51 & 37 & $>300$ & $\mathrm{C}_{21} \mathrm{H}_{20} \mathrm{~N}_{6} \mathrm{~S}_{2}$ & $52.43(52.29)$ & $7.15(7.04)$ & $22.93(22.81)$ & $17.50(17.36)$ \\
\hline $5 \mathrm{~m}$ & 42 & $>300$ & $\mathrm{C}_{22} \mathrm{H}_{22} \mathrm{~N}_{6} \mathrm{~S}_{2}$ & $60.24(60.18)$ & $5.93(5.89)$ & $19.17(19.06)$ & $14.61(14.54)$ \\
\hline $5 n$ & 41 & 175 & $\mathrm{C}_{28} \mathrm{H}_{26} \mathrm{~N}_{6} \mathrm{~S}_{2}$ & $65.85(65.78)$ & $5.13(5.02)$ & $16.46(16.39)$ & $12.56(12.45)$ \\
\hline $\mathbf{8 a}$ & 77 & 295 & $\mathrm{C}_{22} \mathrm{H}_{16} \mathrm{~N}_{6} \mathrm{~S}_{2}$ & $61.70(61.58)$ & $3.69(3.55)$ & 19.68 (19.56) & $14.93(14.82)$ \\
\hline $8 b$ & 73 & 285 & $\mathrm{C}_{24} \mathrm{H}_{20} \mathrm{~N}_{6} \mathrm{~S}_{2}$ & $63.10(63.00)$ & 4.45 (4.34) & $18.39(18.27)$ & $14.06(13.98)$ \\
\hline $9 \mathbf{a}$ & 43 & 162 & $\mathrm{C}_{24} \mathrm{H}_{18} \mathrm{~N}_{6} \mathrm{~S}_{2}$ & $63.40(63.29)$ & $3.99(3.85)$ & $18.56(18.44)$ & $14.05(13.91)$ \\
\hline $9 b$ & 42 & 286 & $\mathrm{C}_{25} \mathrm{H}_{20} \mathrm{~N}_{6} \mathrm{~S}_{2}$ & $64.08(64.00)$ & $4.29(4.19)$ & $18.01(17.90)$ & $13.62(13.50)$ \\
\hline $9 c$ & 45 & 154 & $\mathrm{C}_{26} \mathrm{H}_{22} \mathrm{~N}_{6} \mathrm{~S}_{2}$ & $64.69(64.54)$ & $4.60(4.50)$ & $17.38(17.30)$ & $13.33(13.24)$ \\
\hline 9d & 48 & 270 & $\mathrm{C}_{27} \mathrm{H}_{24} \mathrm{~N}_{6} \mathrm{~S}_{2}$ & $65.29(65.20)$ & $4.78(4.70)$ & $17.02(16.88)$ & $12.91(12.80)$ \\
\hline $9 e$ & 40 & 152 & $\mathrm{C}_{26} \mathrm{H}_{22} \mathrm{~N}_{6} \mathrm{~S}_{2}$ & $64.69(64.55)$ & $4.60(4.48)$ & $17.45(17.34)$ & $13.26(13.14)$ \\
\hline 9f & 45 & 135 & $\mathrm{C}_{27} \mathrm{H}_{24} \mathrm{~N}_{6} \mathrm{~S}_{2}$ & $65.29(65.18)$ & $4.86(4.74)$ & $16.96(16.87)$ & $12.87(12.77)$ \\
\hline $9 \mathrm{~g}$ & 40 & 140 & $\mathrm{C}_{28} \mathrm{H}_{26} \mathrm{~N}_{6} \mathrm{~S}_{2}$ & $65.91(65.80)$ & $5.08(5.00)$ & $16.45(16.33)$ & $12.56(12.45)$ \\
\hline $9 h$ & 45 & $>300$ & $\mathrm{C}_{29} \mathrm{H}_{28} \mathrm{~N}_{6} \mathrm{~S}_{2}$ & $66.38(66.30)$ & $5.36(5.28)$ & $15.98(15.88)$ & $12.27(12.15)$ \\
\hline $9 \mathbf{i}$ & 45 & 280 & $\mathrm{C}_{30} \mathrm{H}_{22} \mathrm{~N}_{6} \mathrm{~S}_{2}$ & $67.90(67.77)$ & $4.16(4.09)$ & $15.88(15.75)$ & $12.06(11.98)$ \\
\hline $\mathbf{9 j}$ & 57 & 285 & $\mathrm{C}_{32} \mathrm{H}_{26} \mathrm{~N}_{6} \mathrm{~S}_{2}$ & $68.80(68.70)$ & $4.70(4.66)$ & $15.11(15.00)$ & $11.39(11.28)$ \\
\hline $9 k$ & 42 & 120 & $\mathrm{C}_{26} \mathrm{H}_{22} \mathrm{~N}_{6} \mathrm{~S}_{2} \mathrm{O}$ & $62.68(62.55)$ & $4.40(4.28)$ & $16.90(16.77)$ & $12.81(12.70)$ \\
\hline 91 & 59 & 105 & $\mathrm{C}_{28} \mathrm{H}_{26} \mathrm{~N}_{6} \mathrm{~S}_{2} \mathrm{O}$ & $63.85(63.78)$ & $4.98(4.86)$ & $16.02(15.95)$ & $12.11(12.00)$ \\
\hline 11 & 85 & 184 & $\mathrm{C}_{16} \mathrm{H}_{20} \mathrm{~N}_{6} \mathrm{~S}_{2} \mathrm{O}_{2}$ & $48.96(48.86)$ & $5.14(5.00)$ & $21.41(21.30)$ & $16.34(16.20)$ \\
\hline $12 a$ & 37 & 119 & $\mathrm{C}_{17} \mathrm{H}_{20} \mathrm{~N}_{6} \mathrm{~S}_{2} \mathrm{O}_{2}$ & $50.48(50.33)$ & $4.98(4.84)$ & $20.78(20.66)$ & $15.85(15.72)$ \\
\hline $12 \mathrm{~b}$ & 38 & 195 & $\mathrm{C}_{18} \mathrm{H}_{22} \mathrm{~N}_{6} \mathrm{~S}_{2} \mathrm{O}_{2}$ & $51.65(51.50)$ & $5.30(5.15)$ & $20.08(20.00)$ & $15.32(15.22)$ \\
\hline $12 \mathrm{c}$ & 38 & 188 & $\mathrm{C}_{19} \mathrm{H}_{24} \mathrm{~N}_{6} \mathrm{~S}_{2} \mathrm{O}_{2}$ & $52.76(52.60)$ & $5.59(5.44)$ & $19.43(19.31)$ & $14.48(14.39)$ \\
\hline $12 d$ & 35 & 160 & $\mathrm{C}_{20} \mathrm{H}_{26} \mathrm{~N}_{6} \mathrm{~S}_{2} \mathrm{O}_{2}$ & $53.79(53.64)$ & $5.87(5.72)$ & $18.82(18.72)$ & $14.36(14.23)$ \\
\hline $12 \mathrm{e}$ & 37 & 142 & $\mathrm{C}_{21} \mathrm{H}_{28} \mathrm{~N}_{6} \mathrm{~S}_{2} \mathrm{O}_{2}$ & $54.76(54.61)$ & $6.13(6.00)$ & $18.25(18.13)$ & $13.92(13.79)$ \\
\hline $12 f$ & 36 & 133 & $\mathrm{C}_{24} \mathrm{H}_{26} \mathrm{~N}_{6} \mathrm{O}_{2} \mathrm{~S}_{2}$ & $58.28(58.12)$ & $5.30(5.15)$ & $16.99(16.88)$ & $12.97(12.83)$ \\
\hline 14 & 75 & 224 & $\mathrm{C}_{14} \mathrm{H}_{12} \mathrm{~N}_{4} \mathrm{SO}$ & $59.14(59.01)$ & 4.25 (4.09) & $19.70(19.57)$ & $11.28(11.13)$ \\
\hline 15 & 63 & 182 & $\mathrm{C}_{31} \mathrm{H}_{28} \mathrm{~N}_{8} \mathrm{~S}_{2} \mathrm{O}_{2}$ & $61.16(61.07)$ & $4.64(4.50)$ & $18.41(18.33)$ & $10.54(10.42)$ \\
\hline $16 a$ & 30 & 168 & $\mathrm{C}_{34} \mathrm{H}_{32} \mathrm{~N}_{8} \mathrm{~S}_{2} \mathrm{O}_{2}$ & $62.94(62.82)$ & $4.97(4.83)$ & $17.27(17.15)$ & $9.88(9.79)$ \\
\hline $16 \mathrm{~b}$ & 39 & 159 & $\mathrm{C}_{35} \mathrm{H}_{34} \mathrm{~N}_{8} \mathrm{O}_{2} \mathrm{~S}_{2}$ & $63.42(63.31)$ & $5.17(5.05)$ & $16.91(16.78)$ & $9.68(9.55)$ \\
\hline $16 \mathrm{c}$ & 37 & 142 & $\mathrm{C}_{39} \mathrm{H}_{34} \mathrm{~N}_{8} \mathrm{~S}_{2} \mathrm{O}_{2}$ & $65.81(65.68)$ & $4.82(4.69)$ & $15.76(15.65)$ & $9.02(8.90)$ \\
\hline
\end{tabular}




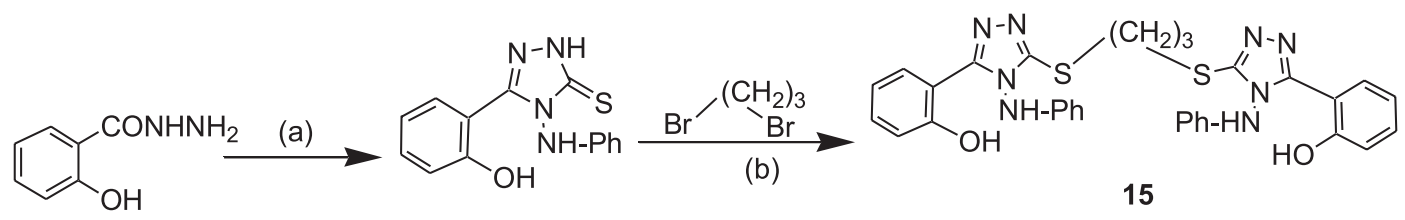

13
14

16a: $m=3$

16b: $m=4$

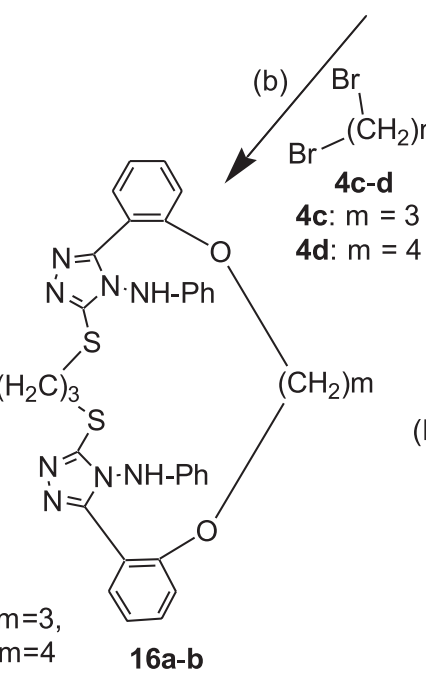

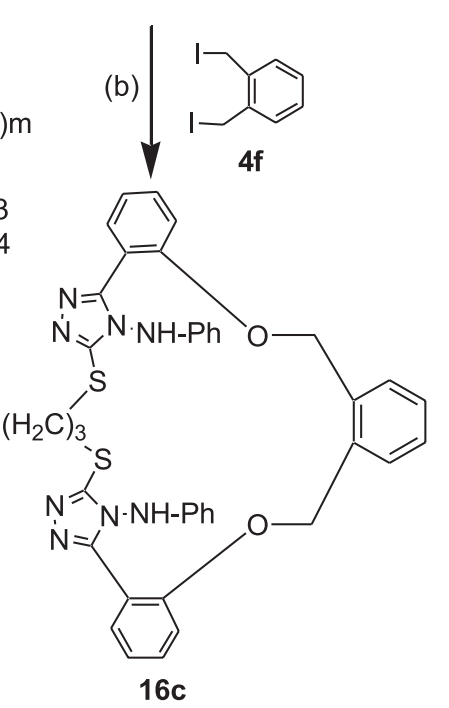

Reagents and conditions: (a) (i) $\mathrm{CS}_{2}, \mathrm{KOH}, \mathrm{MeOH}, 0-5{ }^{\circ} \mathrm{C}$, (ii) $\mathrm{PhNHNH}_{2}$, fusion, $140{ }^{\circ} \mathrm{C}$; (b) $\mathrm{KOH}$, aq. $\mathrm{MeOH}(80 \%), 80{ }^{\circ} \mathrm{C}$.

Scheme 4. Synthesis of benzotriazolophanes 16.

To further explore the chelating properties of benzotriazolophanes, it was decided to incorporate "-O-“" linkage in the heterophane skeleton. Hence, in continuation to this work on benzotriazolophanes, 3-carboxymethoxy phenoxyacetic acid $\mathbf{1 0}$ was fused with 4-ethyl thiosemicarbazide 2a to give compound 11 in high yield (Scheme 3). When 11 was reacted with 1,3-diiodopropane $\mathbf{4 c}$ in methanolic potassium hydroxide, compound 12c was afforded chemoselectively in moderate yield (Scheme 3, Table 1). Structure of the product was confirmed on the basis of spectral data and elemental analysis (Table 1). ${ }^{1} \mathrm{H}$ NMR spectrum of the compound showed triplet at $\delta 3.62$ for $\mathrm{S}-\mathrm{CH}_{2}$ group of propane chain and quintet at $\delta 3.02$ for the central propyl- $\mathrm{CH}_{2}$. The other thiatriazolophanes 12a-f were similarly synthesized and characterized.

We have discussed in detail the chemoselective $\mathrm{N}$-alkylation ${ }^{22}$ and S-alkylation ${ }^{21,22}$ for the synthesis of triazolophanes. In continuation to our work on O-alkylation for the synthesis of heterophanes with amino side arms, ${ }^{22}$ we now report the synthesis of novel N-substituted benzotriazolophanes. Salicylic acid hydrazide 13 was reacted with phenyl hydrazine, carbon disulphide and potassium hydroxide in methanol to give compound 14 in high yield (Scheme 4). When 14 was reacted with 1,3-diiodopropane in methanolic potassium hydroxide, compound $\mathbf{1 5}$ was afforded chemoselectively in good yield (Scheme 4, Table 1). Benzotriazolophanes 16a-c were subsequently synthesized in moderate yield by reacting 15 with diiodoalkanes in aqueous methanol (80\%) containing potassium hydroxide (Scheme 4, Table 1). Structures of the products were confirmed on the basis of spectral data and elemental analysis (Table 1). For example, reaction of $\mathbf{1 5}$ with 1,3-diiodopropane resulted in the formation of the desired compound 16a, which was confirmed on the basis of NMR spectra. ${ }^{1} \mathrm{H}$ NMR spectrum of the compound showed triplet at $\delta 3.62$ for $-\mathrm{SCH}_{2}$ group of propane chain and triplet at $\delta 4.18$ for the propyl $-\mathrm{OCH}_{2}$ groups. $\mathrm{M}^{+}$peak at $\mathrm{m} / \mathrm{z} 648$ in mass spectrum confirmed the formation of $\mathbf{1 6} \mathbf{a}$.

\section{Conclusion}

In this paper, we have reported a versatile and convenient route for the synthesis of novel $\mathrm{N}$-alkyl/aryl thiatriazolophanes from aliphatic and aromatic acids.

\section{Experimental}

The NMR spectra were recorded on Bruker AMX 500 spectrometer at $25^{\circ} \mathrm{C}$. Melting points were taken in open capillaries and are uncorrected. Mass spectra were recorded on Shimadzu GC-MS instrument. 
General procedure for the synthesis of bis-(4-alkyl/aryl-5mercapto-1,2,4-triazol-3-yl)butane (3a-c)

$0.01 \mathrm{~mol}$ of adipic acid and $0.02 \mathrm{~mol}$ of alkyl/aryl thiosemicarbazide were mixed together using mortar and pestle. This mixture was fused on oil-bath at $140{ }^{\circ} \mathrm{C}$ for $4 \mathrm{~h}$, and then cooled to room temperature and the obtained solid mass was dumped into ice-cold water. Brown colored mass was separated by filteration and washed thoroughly with water. Then it dissolved in aqueous $10 \%$ sodium hydroxide solution and the insoluble impurities were removed by filtration. The filtrate was then acidified using aqueous $1 \mathrm{~mol} \mathrm{~L}^{-1} \mathrm{HCl}$ till the $\mathrm{pH}$ of the solution became 2. Separated white colored product was filtered, washed with water and dried under vacuum.

\section{Bis-(4-ethyl-5-mercapto-1,2,4-triazol-3-yl)butane (3a)}

${ }^{1} \mathrm{H}$ NMR, (DMSO d $): \delta 13.51\left(\mathrm{~s}, 2 \mathrm{H}, 2 \mathrm{NH}, \mathrm{D}_{2} \mathrm{O}\right.$ exchangeable), $3.92\left(\mathrm{q}, 4 \mathrm{H}, 2 \mathrm{NCH}_{2}\right), 2.70\left(\mathrm{t}, 4 \mathrm{H}, 2 \mathrm{CH}_{2}\right)$, $1.91\left(\mathrm{p}, 4 \mathrm{H}, 2 \mathrm{CH}_{2}\right), 1.16\left(\mathrm{t}, 6 \mathrm{H}, 2 \mathrm{CH}_{3}\right) \cdot{ }^{13} \mathrm{C} \mathrm{NMR}$ (DMSO $\left.\mathrm{d}_{6}\right): \delta 165.0(2 \underline{\mathrm{C}}=\mathrm{S}), 155.4(2 \underline{\mathrm{C}}=\mathrm{N}), 39.2\left(2 \mathrm{~N}^{\mathrm{CH}}{ }_{2}\right), 25.9$ $\left(2 \underline{\mathrm{CH}}_{2}\right), 24.0\left(2 \underline{\mathrm{CH}}_{2}\right), 15.7\left(2 \underline{\mathrm{CH}}_{3}\right)$.

Bis-(4-allyl-5-mercapto-1,2,4-triazol-3-yl)butane (3b)

${ }^{1} \mathrm{H}$ NMR (DMSO d $\left.\mathrm{d}_{6}\right): \delta 13.60\left(\mathrm{~s}, 2 \mathrm{H}, 2 \mathrm{NH}, \mathrm{D}_{2} \mathrm{O}\right.$ exchangeable), $5.87(\mathrm{~m}, 2 \mathrm{H}, 2=\mathrm{CH}), 5.17,4.98(\mathrm{dd}, 4 \mathrm{H}$, $\left.2=\mathrm{CH}_{2}\right), 4.61\left(\mathrm{~d}, 4 \mathrm{H}, 2 \mathrm{NCH}_{2}\right), 2.51\left(\mathrm{t}, 4 \mathrm{H}, 2 \mathrm{CH}_{2}\right), 1.72(\mathrm{p}$, $\left.4 \mathrm{H}, 2 \mathrm{CH}_{2}\right)$.

Bis-(4-phenyl-5-mercapto-1,2,4-triazol-3-yl)butane (3c)

${ }^{1} \mathrm{H}$ NMR (DMSO d $): \delta 13.60\left(\mathrm{~s}, 2 \mathrm{H}, \mathrm{NH}, \mathrm{D}_{2} \mathrm{O}\right.$ exchangeable), 7.55-7.33 (m, 10H, Aromatic H), $2.32(\mathrm{t}$, $\left.2 \mathrm{H}, 2 \mathrm{CH}_{2}\right), 1.38\left(\mathrm{p}, 4 \mathrm{H}, 2 \mathrm{CH}_{2}\right) \cdot{ }^{13} \mathrm{C} \mathrm{NMR}\left(\mathrm{DMSO} \mathrm{d}_{6}\right): \delta$ $167.6(2 \underline{\mathrm{C}}=\mathrm{S}), 151.7(2 \underline{\mathrm{C}}=\mathrm{N}), 133.6-128.2$ (12 Aromatic C), $24.6\left(2 \underline{\mathrm{CH}}_{2}\right), 24.1\left(2 \underline{\mathrm{CH}}_{2}\right)$.

General procedure for the synthesis of $5 a-n, 8 a-l, 12 a-f$, 15 and $16 a-c$

Compounds 3a-c, 7a-b, 11, 14 and 15 (respectively for the synthesis of 5a-n, 8a-1, 12a-f, 15 and 16a-c) $(0.01 \mathrm{~mol})$ were dissolved in aqueous methanol (80:20, methanol:water; $200 \mathrm{~mL}$ ) containing potassium hydroxide $(0.02 \mathrm{~mol})$. A solution of $1, \omega$-dihaloalkane $(0.01 \mathrm{~mol})$ in methanol was added dropwise for an hour. This reaction mixture was then refluxed with stirring on a magnetic stirrer for eight hours. On cooling it to $10-15{ }^{\circ} \mathrm{C}$, a solid separated out. This solid was then separated by filtration, washed with cold water and recrystallised from aqueous dimethyl formamide (DMF).
$1^{4}, 6^{4}$-Diethyl-7,9-dithia-1,6(3,5)-di-(1,2,4-triazola) cyclononaphane $(\mathbf{5 a})$

${ }^{1} \mathrm{H}$ NMR $\left(\mathrm{CDCl}_{3}\right): \delta 4.70\left(\mathrm{~s}, 2 \mathrm{H},-\mathrm{CH}_{2}\right), 3.91(\mathrm{q}, 4 \mathrm{H}$, $\left.2 \mathrm{NCH}_{2}\right), 2.61\left(\mathrm{t}, 4 \mathrm{H}, 2 \mathrm{CH}_{2}\right), 2.10\left(\mathrm{p}, 4 \mathrm{H}, 2 \mathrm{CH}_{2}\right), 1.16(\mathrm{t}$, $\left.6 \mathrm{H}, 2 \mathrm{CH}_{3}\right) \cdot{ }^{13} \mathrm{C} \mathrm{NMR}\left(\mathrm{DMSO}_{6}\right): \delta 155.6(2 \mathrm{~S}-\mathrm{C}=\mathrm{N}), 146.6$ $\left(2 \mathrm{CH}_{2}-\underline{\mathrm{C}}=\mathrm{N}\right), 40.7\left(\underline{\mathrm{CH}}_{2}\right), 39.2\left(2 \mathrm{NCH}_{2}\right), 25.9\left(2 \underline{\mathrm{CH}}_{2}\right), 25.0$ $\left(2 \underline{\mathrm{CH}}_{2}\right), 15.7\left(2 \mathrm{CH}_{3}\right)$.

$1^{4}, 6^{4}$-Diethyl-7,10-dithia-1,6(3,5)-di-(1,2,4-triazola) cyclodecaphane $(\mathbf{5 b})$

${ }^{1} \mathrm{H} \mathrm{NMR}\left(\mathrm{CDCl}_{3}\right): \delta 4.10\left(\mathrm{t}, 4 \mathrm{H}, 2 \mathrm{SCH}_{2}\right), 3.76(\mathrm{q}, 4 \mathrm{H}$, $\left.2 \mathrm{NCH}_{2}\right), 3.03\left(\mathrm{t}, 4 \mathrm{H}, 2 \mathrm{CH}_{2}\right), 2.49\left(\mathrm{p}, 4 \mathrm{H}, 2 \mathrm{CH}_{2}\right), 1.30$ (t, $\left.6 \mathrm{H}, 2 \mathrm{CH}_{3}\right) .{ }^{13} \mathrm{C} \mathrm{NMR}\left(\mathrm{DMSO}_{6}\right): \delta 156.3(2 \mathrm{~S}-\mathrm{C}=\mathrm{N}), 147.0$ $\left(2 \mathrm{CH}_{2}-\underline{\mathrm{C}}=\mathrm{N}\right), 39.1\left(2 \mathrm{NCH}_{2}\right), 32.1\left(1 \underline{\mathrm{SCH}}_{2}\right), 24.7\left(2 \underline{\mathrm{CH}}_{2}\right)$, $23.1\left(2 \underline{\mathrm{CH}}_{2}\right), 15.3\left(2 \mathrm{CH}_{3}\right)$. MS (DI) $(\mathrm{m} / \mathrm{z}): 338\left(\mathrm{M}^{+}\right)$.

$1^{4}, 6^{4}$-Diethyl-7,11-dithia-1,6(3,5)-di-(1,2,4-triazola) cycloundecaphane $(\mathbf{5 c})$

${ }^{1} \mathrm{H}$ NMR $\left(\mathrm{CDCl}_{3}\right): \delta 3.87\left(\mathrm{q}, 4 \mathrm{H}, 2 \mathrm{NCH}_{2}\right), 3.31(\mathrm{t}, 4 \mathrm{H}$, $\left.2 \mathrm{SCH}_{2}\right), 2.76\left(\mathrm{t}, 4 \mathrm{H}, 2 \mathrm{CH}_{2}\right), 2.10\left(\mathrm{p}, 2 \mathrm{H}, 1 \mathrm{CH}_{2}\right), 1.95$ (p, 4H, $\left.2 \mathrm{CH}_{2}\right), 1.30\left(\mathrm{t}, 6 \mathrm{H}, 2 \mathrm{CH}_{3}\right) \cdot{ }^{13} \mathrm{C}$ NMR (DMSO d $): \delta 153.5$ $(2 \mathrm{~S}-\mathrm{C}=\mathrm{N}), 149.6\left(2 \mathrm{CH}_{2}-\mathrm{C}=\mathrm{N}\right), 39.8\left(2 \mathrm{NCH}_{2}\right), 36.2\left(2 \mathrm{SCH}_{2}\right)$, $28.2\left(\underline{\mathrm{CH}}_{2}\right), 25.9\left(2 \underline{\mathrm{CH}}_{2}\right), 24.3\left(2 \mathrm{CH}_{2}\right), 15.4\left(2 \underline{\mathrm{CH}}_{3}\right)$.

$1^{4}, 6^{4}$-Diethyl-7,12-dithia-1,6(3,5)-di-(1,2,4-triazola) cyclododecaphane $(\mathbf{5 d})$

${ }^{1} \mathrm{H}$ NMR $\left(\mathrm{CDCl}_{3}\right): \delta 3.86\left(\mathrm{q}, 4 \mathrm{H}, 2 \mathrm{NCH}_{2}\right), 3.20(\mathrm{t}$, $\left.4 \mathrm{H}, 2 \mathrm{SCH}_{2}\right), 2.72$ (t, 4H, 2CH$), 2.03\left(\mathrm{p}, 4 \mathrm{H}, 2 \mathrm{CH}_{2}\right), 1.84$ (p, 4H, 2 $\left.\mathrm{CH}_{2}\right), 1.28\left(\mathrm{t}, 6 \mathrm{H}, 2 \mathrm{CH}_{3}\right) .{ }^{13} \mathrm{C} \mathrm{NMR}\left(\mathrm{DMSO} \mathrm{d}_{6}\right)$ : $\delta 154.8(2 \mathrm{~S}-\underline{\mathrm{C}}=\mathrm{N}), 149.7\left(2 \mathrm{CH}_{2}-\underline{\mathrm{C}}=\mathrm{N}\right), 38.6\left(2 \mathrm{NCH}_{2}\right)$, $32.5\left(2 \mathrm{SCH}_{2}\right), 28.4\left(2 \underline{\mathrm{CH}}_{2}\right), 26.3\left(2 \underline{\mathrm{CH}}_{2}\right), 24.6\left(2 \underline{\mathrm{CH}}_{2}\right)$, $15.3\left(2 \mathrm{CH}_{3}\right)$.

$1^{4}, 6^{4}$-Diethyl-7,13-dithia-1,6(3,5)-di-(1,2,4-triazola) cyclotridecaphane (5e)

${ }^{1} \mathrm{H}$ NMR $\left(\mathrm{CDCl}_{3}\right): \delta 3.87\left(\mathrm{q}, 4 \mathrm{H}, 2 \mathrm{NCH}_{2}\right), 3.19(\mathrm{t}, 4 \mathrm{H}$, $\left.2 \mathrm{SCH}_{2}\right), 2.76\left(\mathrm{t}, 4 \mathrm{H}, 2 \mathrm{CH}_{2}\right), 1.94\left(\mathrm{p}, 4 \mathrm{H}, 2 \mathrm{CH}_{2}\right), 1.75(\mathrm{p}, 4 \mathrm{H}$, $\left.2 \mathrm{CH}_{2}\right), 1.52\left(\mathrm{p}, 2 \mathrm{H}, 1 \mathrm{CH}_{2}\right), 1.25\left(\mathrm{t}, 6 \mathrm{H}, 2 \mathrm{CH}_{3}\right) .{ }^{13} \mathrm{C} \mathrm{NMR}$ $\left(\right.$ DMSO d $\left._{6}\right): \delta 154.8(2 \mathrm{~S}-\underline{\mathrm{C}}=\mathrm{N}), 149.9\left(2 \mathrm{CH}_{2}-\underline{\mathrm{C}}=\mathrm{N}\right), 38.6$ $\left(2 \mathrm{NCH}_{2}\right), 32.9\left(2 \mathrm{SCH}_{2}\right), 28.2\left(2 \underline{\mathrm{CH}}_{2}\right), 26.3\left(2 \underline{\mathrm{CH}}_{2}\right), 24.6$ $\left(1 \underline{\mathrm{CH}}_{2}\right), 24.1\left(2 \underline{\mathrm{CH}}_{2}\right), 15.3\left(2 \underline{\mathrm{CH}}_{3}\right)$.

$1^{4}, 6^{4}$-Diallyl-7,9-dithia-1,6(3,5)-di-(1,2,4-triazola) cyclononaphane $(\mathbf{5 f})$

${ }^{1} \mathrm{H}$ NMR (DMSO d $)$ ): $\delta 5.80(\mathrm{~m}, 2 \mathrm{H}, 2=\mathrm{CH}), 5.21(\mathrm{dd}$, $\left.4 \mathrm{H}, 2=\mathrm{CH}_{2}\right), 4.73\left(\mathrm{~s}, 2 \mathrm{H}, 1 \mathrm{CH}_{2}\right), 4.69\left(\mathrm{~d}, 4 \mathrm{H}, 2 \mathrm{NCH}_{2}\right)$, $2.58\left(\mathrm{t}, 4 \mathrm{H}, 2 \mathrm{CH}_{2}\right), 2.10\left(\mathrm{p}, 4 \mathrm{H}, 2 \mathrm{CH}_{2}\right) .{ }^{13} \mathrm{C} \mathrm{NMR}$ (DMSO $\left.\mathrm{d}_{6}\right): \delta 155.9(2 \mathrm{~S}-\underline{\mathrm{C}}=\mathrm{N}), 147.4(2 \mathrm{C}-\underline{\mathrm{C}}=\mathrm{N}), 131.0(2 \mathrm{C}=\underline{\mathrm{CH}})$, $118.3\left(2 \mathrm{C}=\underline{\mathrm{CH}}_{2}\right), 46.1\left(\mathrm{SCH}_{2}\right), 40.7\left(2 \mathrm{NCH}_{2}\right), 25.4\left(2 \underline{\mathrm{CH}}_{2}\right)$, $21.8\left(2 \mathrm{CH}_{2}\right)$. 
$1^{4}, 6^{4}$-Diallyl-7,11-dithia-1,6(3,5)-di-(1,2,4-triazola) cycloundecaphane $\mathbf{( 5 g})$

${ }^{1} \mathrm{H}$ NMR (DMSO d $): \delta 5.81(\mathrm{~m}, 2 \mathrm{H}, 2=\mathrm{CH}), 5.19(\mathrm{dd}$, $\left.4 \mathrm{H}, 2=\mathrm{CH}_{2}\right), 4.55\left(\mathrm{~d}, 4 \mathrm{H}, 2 \mathrm{NCH}_{2}\right), 3.24\left(\mathrm{t}, 4 \mathrm{H}, 2 \mathrm{CH}_{2}\right)$,

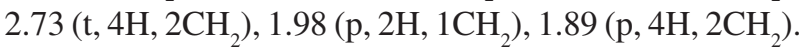
${ }^{13} \mathrm{C}$ NMR $\left(\operatorname{DMSO}_{6}\right): \delta 155.4(2 \mathrm{~S}-\mathrm{C}=\mathrm{N}), 149.4(2-\mathrm{C}-\underline{\mathrm{C}}=\mathrm{N})$, $131.3(2 \mathrm{C}=\underline{\mathrm{CH}}), 117.8\left(2 \mathrm{C}=\underline{\mathrm{CH}}_{2}\right), 45.8\left(2 \underline{\mathrm{CH}}_{2}\right), 32.1$ $\left(2 \mathrm{NCH}_{2}\right), 29.3\left(2 \underline{\mathrm{CH}}_{2}\right), 26.1\left(\underline{\mathrm{CH}}_{2}\right), 24.2\left(2 \underline{\mathrm{CH}}_{2}\right)$.

$1^{4}, 6^{4}$-Diallyl-7,12-dithia-1,6(3,5)-di-(1,2,4-triazola) cyclododecaphane $(\mathbf{5 h})$

${ }^{1} \mathrm{H}$ NMR (DMSO d $): \delta 5.82(\mathrm{~m}, 2 \mathrm{H}, 2=\mathrm{CH}), 5.10(\mathrm{dd}$, $\left.4 \mathrm{H}, 2=\mathrm{CH}_{2}\right), 4.58\left(\mathrm{~d}, 4 \mathrm{H}, 2 \mathrm{NCH}_{2}\right), 3.13\left(\mathrm{t}, 4 \mathrm{H}, 2 \mathrm{CH}_{2}\right), 2.50$ (t, 4H, 2CH $), 2.01\left(\mathrm{p}, 4 \mathrm{H}, 1 \mathrm{CH}_{2}\right), 1.72\left(\mathrm{p}, 4 \mathrm{H}, 2 \mathrm{CH}_{2}\right)$. ${ }^{13} \mathrm{C}$ NMR $\left(\mathrm{DMSO} \mathrm{d}_{6}\right): \delta 155.5(2 \mathrm{~S}-\underline{\mathrm{C}}=\mathrm{N}), 149.1(2 \mathrm{C}-\underline{\mathrm{C}}=\mathrm{N})$, $131.1(2 \mathrm{C}=\underline{\mathrm{CH}}), 117.9\left(2 \mathrm{C}=\underline{\mathrm{CH}}_{2}\right), 45.7\left(2 \mathrm{~S}_{\mathrm{CH}}\right), 32.6$ $\left(2 \mathrm{NCH}_{2}\right), 28.4\left(2 \underline{\mathrm{CH}}_{2}\right), 23.8\left(2 \underline{\mathrm{CH}}_{2}\right), 23.5\left(2 \underline{\mathrm{CH}}_{2}\right)$.

$1^{4}, 6^{4}$-Diallyl-7,13-dithia-1,6(3,5)-di-(1,2,4-triazola) cyclotridecaphane (5i)

${ }^{1} \mathrm{H}$ NMR (DMSO d $)$ ): $\delta 5.82(\mathrm{~m}, 2 \mathrm{H}, 2=\mathrm{CH}), 5.07$ (dd, $\left.4 \mathrm{H}, 2=\mathrm{CH}_{2}\right), 4.24\left(\mathrm{~d}, 4 \mathrm{H}, 2 \mathrm{NCH}_{2}\right), 3.35\left(\mathrm{t}, 4 \mathrm{H}, 2 \mathrm{CH}_{2}\right), 2.66$ $\left(\mathrm{t}, 4 \mathrm{H}, 2 \mathrm{CH}_{2}\right), 1.90\left(\mathrm{p}, 4 \mathrm{H}, 1 \mathrm{CH}_{2}\right), 1.72\left(\mathrm{p}, 4 \mathrm{H}, 2 \mathrm{CH}_{2}\right), 1.58$ $\left(\mathrm{p}, 2 \mathrm{H}, 1 \mathrm{CH}_{2}\right) \cdot{ }^{13} \mathrm{C} \mathrm{NMR}\left(\mathrm{DMSO} \mathrm{d}_{6}\right): \delta 155.2(2 \mathrm{~S}-\mathrm{C}=\mathrm{N})$, $150.4(2 \mathrm{C}-\underline{\mathrm{C}}=\mathrm{N}), 131.8(2 \mathrm{C}=\underline{\mathrm{CH}}), 117.8\left(2 \mathrm{C}=\underline{\mathrm{CH}}_{2}\right), 45.5$ $\left(2 \underline{\mathrm{CH}}_{2}\right), 33.1\left(2 \mathrm{~N}_{\mathrm{CH}}\right), 28.9\left(2 \underline{\mathrm{CH}}_{2}\right), 28.1\left(2 \underline{\mathrm{CH}}_{2}\right), 25.3$ $\left(\underline{\mathrm{CH}}_{2}\right), 24.5\left(2 \underline{\mathrm{CH}}_{2}\right)$.

$1^{4}, 6^{4}$-Diphenyl-7,9-dithia-1,6(3,5)-di-(1,2,4-triazola)cyclononaphane $(\mathbf{5 j})$

${ }^{1} \mathrm{H}$ NMR (DMSO d $)$ ): $\delta 7.52-7.77(\mathrm{~m}, 10 \mathrm{H}$, Aromatic $\mathrm{H}), 4.64\left(\mathrm{~s}, 2 \mathrm{H}, \mathrm{CH}_{2}\right), 2.52\left(\mathrm{t}, 4 \mathrm{H}, 2 \mathrm{CH}_{2}\right), 2.00(\mathrm{p}, 4 \mathrm{H}$, $\left.2 \mathrm{CH}_{2}\right) \cdot{ }^{13} \mathrm{C}$ NMR (DMSO d $): \delta 157.0(2 \mathrm{~S}-\underline{\mathrm{C}}=\mathrm{N}), 148.8$ $(2 \mathrm{C}-\underline{\mathrm{C}}=\mathrm{N}), 133.3-127.6$ (12 Aromatic C), $41.5\left(\underline{\mathrm{CH}}_{2}\right), 25.0$ $\left(2 \underline{\mathrm{CH}}_{2}\right), 22.3\left(2 \underline{\mathrm{CH}}_{2}\right)$.

$1^{4}, 6^{4}$-Diphenyl-7,10-dithia-1,6(3,5)-di-(1,2,4-triazola) cyclodecaphane $(\mathbf{5} \boldsymbol{k})$

${ }^{1} \mathrm{H}$ NMR (DMSO d $): \delta 7.63-7.55(\mathrm{~m}, 10 \mathrm{H}$, Aromatic $\mathrm{H}), 2.86\left(\mathrm{t}, 4 \mathrm{H}, 2 \mathrm{CH}_{2}\right), 2.64\left(\mathrm{t}, 4 \mathrm{H}, 2 \mathrm{CH}_{2}\right), 1.96(\mathrm{p}, 4 \mathrm{H}$, $\left.2 \mathrm{CH}_{2}\right) \cdot{ }^{13} \mathrm{C}$ NMR (DMSO d $): \delta 158.0(2 \mathrm{~S}-\underline{\mathrm{C}}=\mathrm{N}), 148.8$ $(2 \mathrm{C}-\underline{\mathrm{C}}=\mathrm{N}), 133.3-127.9$ (12 Aromatic C), $32.1\left(2 \underline{\mathrm{CH}}_{2}\right)$, $25.1\left(2 \underline{\mathrm{CH}}_{2}\right), 23.1\left(2 \underline{\mathrm{CH}}_{2}\right)$.

$1^{4}, 6^{4}$-Diethyl-9(1,2)-benzena-7,11-dithia-1,3(3,5)(5,3)di-(1,2,4-triazola)cycloundecaphane $(5 \mathrm{l})$

${ }^{1} \mathrm{H}$ NMR $\left(\mathrm{CDCl}_{3}\right)$ : $\delta$ 7.73-6.95 (m, 4H, Aromatic $\left.\mathrm{H}\right)$, $4.72\left(\mathrm{~s}, 4 \mathrm{H}, 2 \mathrm{SCH}_{2}\right), 3.80$ (q, $\left.4 \mathrm{H}, 2 \mathrm{NCH}_{2}\right), 2.76(\mathrm{t}, 4 \mathrm{H}$, $\left.2 \mathrm{CH}_{2}\right), 2.00\left(\mathrm{p}, 4 \mathrm{H}, 2 \mathrm{CH}_{2}\right), 1.17\left(\mathrm{t}, 6 \mathrm{H}, 2 \mathrm{CH}_{3}\right) .{ }^{13} \mathrm{C} \mathrm{NMR}$ $($ DMSO d $): \delta 155.5(2 \mathrm{~S}-\underline{\mathrm{C}}=\mathrm{N}), 149.6(2 \mathrm{C}-\underline{\mathrm{C}}=\mathrm{N}), 133.0-$ 125.0 (6 Aromatic C), $45.7\left(2 \mathrm{SCH}_{2}\right), 36.0\left(2 \mathrm{NCH}_{2}\right), 25.9$ $\left(2 \underline{\mathrm{CH}}_{2}\right), 24.6\left(2 \underline{\mathrm{CH}}_{2}\right), 15.4\left(2 \underline{\mathrm{CH}}_{3}\right)$.

$1^{4}, 6^{4}$-Diallyl-9(1,2)-benzena-7,11-dithia-1,3(3,5)(5,3)-di(1,2,4-triazola)cycloundecaphane (5m)

${ }^{1} \mathrm{H}$ NMR (DMSO $\mathrm{d}_{6}$ ): $\delta$ 7.63-7.04 (m, 4H, Aromatic $\mathrm{H}), 5.74(\mathrm{~m}, 2 \mathrm{H}, 2 \mathrm{C}=\mathrm{CH}), 5.16\left(\mathrm{dd}, 4 \mathrm{H}, 2 \mathrm{C}=\mathrm{CH}_{2}\right), 4.73$ $\left(\mathrm{s}, 4 \mathrm{H}, 2 \mathrm{SCH}_{2}\right), 4.38\left(\mathrm{~d}, 4 \mathrm{H}, 2 \mathrm{NCH}_{2}\right), 2.71\left(\mathrm{t}, 4 \mathrm{H}, 2 \mathrm{CH}_{2}\right)$, $1.72\left(\mathrm{p}, 4 \mathrm{H}, 2 \mathrm{CH}_{2}\right) .{ }^{13} \mathrm{C}$ NMR (DMSO d $): \delta 155.5(2 \mathrm{~S}-$ $\underline{\mathrm{C}}=\mathrm{N}), 149.6(2 \mathrm{C}-\underline{\mathrm{C}}=\mathrm{N}), 133.0-125.0(6$ Aromatic $\mathrm{C}), 131.2$ $(2 \mathrm{C}=\underline{\mathrm{CH}}), 117.8\left(2 \mathrm{C}=\underline{\mathrm{CH}}_{2}\right), 45.7\left(2 \mathrm{~S}_{\mathrm{CH}}\right), 36.0\left(2 \mathrm{~N}_{2} \mathrm{H}_{2}\right)$, $25.9\left(2 \mathrm{CH}_{2}\right), 24.6\left(2 \underline{\mathrm{CH}}_{2}\right)$.

$1^{4}, 6^{4}$-Diphenyl-9(1,2)-benzena-7,11-dithia-1,3(3,5)(5,3)di-(1,2,4-triazola)cycloundecaphane (5n)

${ }^{1} \mathrm{H}$ NMR (DMSO d $\left.)_{6}\right) \delta 7.65-7.15(\mathrm{~m}, 14 \mathrm{H}$, Aromatic $\mathrm{H}), 4.82\left(\mathrm{~s}, 4 \mathrm{H}, 2 \mathrm{SCH}_{2}\right), 2.69\left(\mathrm{t}, 4 \mathrm{H}, 2 \mathrm{CH}_{2}\right), 2.03$ (p, 4H, $\left.2 \mathrm{CH}_{2}\right) \cdot{ }^{13} \mathrm{C}$ NMR (DMSO d $): \delta 158.0(2 \mathrm{~S}-\underline{\mathrm{C}}=\mathrm{N}), 148.8$ $(2 \mathrm{C}-\underline{\mathrm{C}}=\mathrm{N}), 133.3-127.9$ (18 Aromatic C), $38.0\left(2 \underline{\mathrm{S}}_{2}\right)$, $25.1\left(2 \underline{\mathrm{CH}}_{2}\right), 23.2\left(2 \underline{\mathrm{CH}}_{2}\right)$.

$1^{4}, 3^{4}$-Diphenyl-2(1,3)-benzena-4,7-dithia-1,3(3,5)(5,3)di-(1,2,4-triazola)cycloheptaphane (9a)

${ }^{1} \mathrm{H}$ NMR (DMSO d $): \delta 7.58-7.22(\mathrm{~m}, 14 \mathrm{H}$, Aromatic $\mathrm{H}), 3.46\left(\mathrm{t}, 4 \mathrm{H}, 2 \mathrm{SCH}_{2}\right) .{ }^{13} \mathrm{C} \mathrm{NMR}\left(\mathrm{DMSO} \mathrm{d}_{6}\right): \delta 153.6$, $151.3(4 \underline{\mathrm{C}}=\mathrm{N}), 133.4-127.0$ (18x Aromatic C), 31.8 $\left(2 \underline{S C H}_{2}\right) . \mathrm{MS}(\mathrm{DI})(\mathrm{m} / \mathrm{z}): 454\left(\mathrm{M}^{+}\right)$.

14,34-Diphenyl-2(1,3)-benzena-4,8-dithia-1,3(3,5)(5,3)di-(1,2,4-triazola)cycloctaphane $(\mathbf{9 b})$

${ }^{1} \mathrm{H}$ NMR (DMSO d $)$ ): $\delta 7.68-7.28(\mathrm{~m}, 14 \mathrm{H}$, Aromatic H), 3.23 (t, 4H, 2SCH 2$), 1.96\left(\mathrm{p}, 2 \mathrm{H}, \mathrm{CH}_{2}\right)$. MS (DI) $(\mathrm{m} / \mathrm{z})$ : $468\left(\mathrm{M}^{+}\right)$.

$1^{4}, 3^{4}$-Diphenyl-2(1,3)-benzena-4,9-dithia-1,3,(3,5)(5,3)di-(1,2,4-triazola)cyclononaphane $(\mathbf{9 c})$

${ }^{1} \mathrm{H}$ NMR (DMSO d $): \delta$ 7.55-7.19 $(\mathrm{m}, 14 \mathrm{H}$, Aromatic $\mathrm{H}), 3.13$ (t, 4H, $2 \mathrm{SCH}_{2}$ ), 1.73 (p, 4H, $\left.2 \mathrm{CH}_{2}\right) .{ }^{13} \mathrm{C} \mathrm{NMR}$ $\left(\right.$ DMSO d$\left._{6}\right): \delta 153.4,152.0(4 \underline{\mathrm{C}}=\mathrm{N}), 133.5-124.8(18$

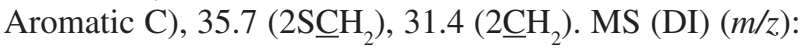
$482\left(\mathrm{M}^{+}\right)$.

$1^{4}, 3^{4}$-Diphenyl-2(1,3)-benzena-4,10-dithia-1,3(3,5)(5,3)di-(1,2,4-triazola)cyclodecaphane $(\mathbf{9 d})$

${ }^{1} \mathrm{H}$ NMR (DMSO d $): \delta 7.51-7.21(\mathrm{~m}, 14 \mathrm{H}$, Aromatic $\mathrm{H}), 3.05$ (t, 4H, 2SCH$\left.)_{2}\right), 1.64$ (p, 4H, 2CH $), 1.33$ (p, 2H, $\left.\mathrm{CH}_{2}\right)$. MS (DI) $(\mathrm{m} / \mathrm{z}): 496\left(\mathrm{M}^{+}\right)$.

$1^{4}, 3^{4}$-Di-[(2'-methyl)phenyl]-2(1,3)6(1,2)-dibenzena-4,7dithia-1(3,5)3(5,3)-di-(1,2,4-triazola)cycloheptaphane (9e)

${ }^{1} \mathrm{H}$ NMR (DMSO d $): \delta 7.63-6.90(\mathrm{~m}, 12 \mathrm{H}$, Aromatic 
$\mathrm{H}), 5.52\left(\mathrm{t}, 4 \mathrm{H}, 2 \mathrm{SCH}_{2}\right), 1.90\left(\mathrm{~s}, 3 \mathrm{H}, \mathrm{CH}_{3}\right), 1.85(\mathrm{~s}, 3 \mathrm{H}$, $\left.\mathrm{CH}_{3}\right) .{ }^{13} \mathrm{C}$ NMR $\left(\mathrm{DMSO} \mathrm{d}_{6}\right): 151.0,149.0(4 \underline{\mathrm{C}}=\mathrm{N}), 135.6-$ 118.7 (18 Aromatic C), 41.9, $31.4\left(2 \underline{S}^{2}{ }_{2}\right), 17.4,17.3$ $\left(2 \underline{\mathrm{CH}}_{3}\right) . \mathrm{MS}(\mathrm{DI})(\mathrm{m} / \mathrm{z}): 482\left(\mathrm{M}^{+}\right)$.

$1^{4}, 3^{4}-D i-[(2 '$-methyl $)$ phenyl $]-2(1,3) 6(1,2)$-dibenzena-4, 8-dithia-1(3,5)3(5,3)-di-(1,2,4-triazola)cycloctaphane (9f)

${ }^{1} \mathrm{H}$ NMR (DMSO d $): \delta 7.32-7.13(\mathrm{~m}, 12 \mathrm{H}$, Aromatic $\mathrm{H}), 3.76\left(\mathrm{t}, 4 \mathrm{H}, 2 \mathrm{SCH}_{2}\right), 2.06\left(\mathrm{p}, 2 \mathrm{H}, \mathrm{CH}_{2}\right), 1.92(\mathrm{~s}, 3 \mathrm{H}$, $\left.\mathrm{CH}_{3}\right), 1.87\left(\mathrm{~s}, 3 \mathrm{H}, \mathrm{CH}_{3}\right) .{ }^{13} \mathrm{C} \mathrm{NMR}\left(\mathrm{DMSO} \mathrm{d}_{6}\right): \delta 153.8$, $152.6(4 \underline{\mathrm{C}}=\mathrm{N}), 135.6-126.5$ (18 Aromatic C), 30.8, 30.6 $\left(2 \underline{\mathrm{CH}}_{2}\right), 28.7\left(\underline{\mathrm{CH}}_{2}\right), 17.7,17.4\left(2 \underline{\mathrm{CH}}_{3}\right) . \mathrm{MS}(\mathrm{DI})(\mathrm{m} / \mathrm{z})$ : $496\left(\mathrm{M}^{+}\right)$.

$1^{4}, 3^{4}-D i-\left[\left(2^{\prime}-m e t h y l\right)\right.$ phenyl $]-2(1,3) 6(1,2)$-dibenzena-4, 9-dithia-1(3,5)3(5,3)-di-(1,2,4-triazola)cyclononaphane (9g)

${ }^{1} \mathrm{H}$ NMR (DMSO d $): \delta 7.80-7.09(\mathrm{~m}, 12 \mathrm{H}$, Aromatic $\mathrm{H}), 3.31\left(\mathrm{t}, 4 \mathrm{H}, 2 \mathrm{SCH}_{2}\right), 1.93\left(\mathrm{~s}, 3 \mathrm{H}, \mathrm{CH}_{3}\right), 1.85(\mathrm{p}, 4 \mathrm{H}$, $\left.2 \mathrm{CH}_{2}\right), 1.80\left(\mathrm{~s}, 3 \mathrm{H}, \mathrm{CH}_{3}\right) \cdot{ }^{13} \mathrm{C} \mathrm{NMR}\left(\mathrm{DMSO} \mathrm{d}_{6}\right): \delta 153.8$, $153.1(4 \underline{\mathrm{C}}=\mathrm{N}), 135.6-127.4$ (18x Aromatic C), 31.4, 31.3 $\left(2 \underline{\mathrm{CH}}_{2}\right), 28.3\left(\underline{\mathrm{CH}}_{2}\right), 17.5,17.4\left(2 \underline{\mathrm{CH}}_{3}\right) . \mathrm{MS}(\mathrm{DI})(\mathrm{m} / \mathrm{z})$ : $510\left(\mathrm{M}^{+}\right)$.

$1^{4}, 3^{4}-$ Di-[(2'-methyl)phenyl $]-2(1,3) 6(1,2)$-dibenzena-4, 10-dithia-1(3,5)3(5,3)-di-(1,2,4-triazola)cyclodecaphane (9h)

${ }^{1} \mathrm{H}$ NMR (DMSO d $): \delta$ 7.60-7.12 $(\mathrm{m}, 12 \mathrm{H}$, Aromatic $\mathrm{H}), 3.21\left(\mathrm{t}, 4 \mathrm{H}, 2 \mathrm{SCH}_{2}\right), 1.91\left(\mathrm{~s}, 3 \mathrm{H}, \mathrm{CH}_{3}\right), 1.89$ (s, 3H, $\left.\mathrm{CH}_{3}\right), 1.68$ (q, 4H, 2CH $\mathrm{CH}_{2}, 1.55$ (p, 2H, $\left.\mathrm{CH}_{2}\right) .{ }^{13} \mathrm{C} \mathrm{NMR}$ $\left(\right.$ DMSO d $\left._{6}\right): \delta 153.8,153.2(4 \underline{\mathrm{C}}=\mathrm{N}), 135.5-127.4(18$ Aromatic C), 32.2, $31.8\left(2 \underline{S}^{-} H_{2}\right), 28.7,27.2\left(3 \underline{\mathrm{CH}}_{2}\right), 17.3$ $\left(\underline{\mathrm{CH}}_{3}\right) . \mathrm{MS}(\mathrm{DI})(\mathrm{m} / \mathrm{z}): 524\left(\mathrm{M}^{+}\right)$.

\section{$1^{4}, 3^{4}$-Diphenyl-2(1,3)6(1,2)-dibenzena-4,8-dithia-1,3(3,5) (5,3)-di-(1,2,4-triazola)cycloctaphane (9i) \\ ${ }^{1} \mathrm{H}$ NMR (DMSO d $)$ ) $7.57-7.05(\mathrm{~m}, 14 \mathrm{H}$, Aromatic H), $4.53\left(\mathrm{~s}, 4 \mathrm{H}, 2 \mathrm{SCH}_{2}\right) \cdot{ }^{13} \mathrm{C}$ NMR (DMSO d 6 ): $\delta 154.4,152.4$ $(4 \underline{\mathrm{C}}=\mathrm{N}), 135.9-127.8$ (24 Aromatic C), $34.7\left(2 \mathrm{~S}^{\mathrm{CH}}{ }_{2}\right) . \mathrm{MS}$ (DI) $(\mathrm{m} / z): 530\left(\mathbf{M}^{+}\right)$.}

$1^{4}, 3^{4}-D i-[(2-m e t h y l)$ phenyl $\left.)\right]-2(1,3) 6(1,2)$-dibenzena-4, 8-dithia-1(3,5)3(5,3)-di-(1,2,4-triazola)cycloctaphane (9j)

${ }^{1} \mathrm{H}$ NMR $\left(\mathrm{CDCl}_{3}\right) \delta$ 7.23-6.95 (m, 12H, Aromatic $\mathrm{H}), 4.76\left(\mathrm{~s}, 2 \mathrm{H}, \mathrm{SCH}_{2}\right), 4.40\left(\mathrm{~s}, 2 \mathrm{H}, \mathrm{SCH}_{2}\right), 1.96(\mathrm{~s}, 3 \mathrm{H}$, $\left.\mathrm{CH}_{3}\right), 1.89$ (s, $\left.3 \mathrm{H}, \mathrm{CH}_{3}\right) \cdot{ }^{13} \mathrm{C} \mathrm{NMR}\left(\mathrm{CDCl}_{3}\right): \delta 152.9,151.3$ $(4 \underline{\mathrm{C}}=\mathrm{N}), 134.4-126.3$ (18 Aromatic C), 33.4, 33.1, $\left(2 \mathrm{SCH}_{2}\right)$, $16.5,16.2\left(2 \underline{\mathrm{CH}}_{3}\right)$. MS (DI) $(\mathrm{m} / \mathrm{z}): 558\left(\mathrm{M}^{+}\right)$.
$1^{4}, 3^{4}$-Diphenyl-2(1,3)-benzena-7-oxa-4,10-dithia-1, 3(3,5)(5,3)-di-(1,2,4-triazola)cyclodecaphane (9k)

${ }^{1} \mathrm{H}$ NMR (DMSO d $): \delta 7.51-7.22(\mathrm{~m}, 14 \mathrm{H}$, Aromatic $\mathrm{H}), 3.70\left(\mathrm{t} 4 \mathrm{H}, 2 \mathrm{OCH}_{2}\right), 3.64\left(\mathrm{t} 4 \mathrm{H}, 2 \mathrm{SCH}_{2}\right) .{ }^{13} \mathrm{C} \mathrm{NMR}$ $\left(\right.$ DMSO d $\left._{6}\right): \delta 153.5,152.0(4 \underline{\mathrm{C}}=\mathrm{N}), 133.5-127.0$ (18 Aromatic C), $70.2\left(2 \mathrm{OCH}_{2}\right), 43.4\left(2 \underline{\mathrm{C} H}_{2}\right) . \mathrm{MS}(\mathrm{DI})(\mathrm{m} / \mathrm{z})$ : $498\left(\mathrm{M}^{+}\right)$.

$1^{4}, 3^{4}-D i-\left[\left(2^{\prime}-\right.\right.$ methyl)phenyl $]-2(1,3) 6(1,2)$-dibenzena-7oxa-4,10-dithia-1(3,5)3(5,3)-di-(1,2,4-triazola)cyclodecaphane (9l)

${ }^{1} \mathrm{H}$ NMR (DMSO d $): \delta 7.59-7.10(\mathrm{~m}, 16 \mathrm{H}$, Aromatic $\mathrm{H}), 3.90-3.44\left(\mathrm{~m}, 8 \mathrm{H}, 2 \mathrm{OCH}_{2} \& 2 \mathrm{SCH}_{2}\right), 1.87(\mathrm{~s}, 3 \mathrm{H}$, $\left.-\mathrm{CH}_{3}\right), 1.85\left(\mathrm{~s}, 3 \mathrm{H},-\mathrm{CH}_{3}\right) .{ }^{13} \mathrm{C}$ NMR $\left(\mathrm{DMSO} \mathrm{d}_{6}\right): \delta 153.8$, $152.9(4 \underline{\mathrm{C}}=\mathrm{N}), 135.5-126.4$ (24 Aromatic C), 70.1, 69.1 $\left(2 \underline{\mathrm{CH}}_{2}\right), 42.6,31.4\left(2 \underline{\mathrm{S}}_{2}\right), 17.4,17.3\left(2 \underline{\mathrm{CH}}_{3}\right) . \mathrm{MS}$ (DI) $(\mathrm{m} / \mathrm{z}): 526\left(\mathbf{M}^{+}\right)$.

$1^{4} 5^{4}$-Diethyl-3(1,3)-benzena-2,4-dioxa-6,8-dithia-1,5(3,5) (5,3)-di-(1,2,4-triazola)cycloctaphane (12a)

${ }^{1} \mathrm{H}$ NMR $\left(\mathrm{CDCl}_{3}\right): \delta$ 7.25-6.64 $(\mathrm{m}, 4 \mathrm{H}$, Aromatic $\mathrm{H})$, $5.10\left(\mathrm{~s}, 4 \mathrm{H}, 2 \mathrm{OCH}_{2}\right), 4.70\left(\mathrm{~s}, 2 \mathrm{H}, 1 \mathrm{CH}_{2}\right), 4.02(\mathrm{q}, 4 \mathrm{H}$, $\left.2 \mathrm{NCH}_{2}\right), 1.26\left(\mathrm{t}, 6 \mathrm{H}, 2 \mathrm{CH}_{3}\right) \cdot{ }^{13} \mathrm{C} \mathrm{NMR}\left(\mathrm{CDCl}_{3}\right): \delta 152.5$, $147.8(4 \underline{\mathrm{C}}=\mathrm{N}), 158.6,112.4,107.9,102.1$ (6 Aromatic $\mathrm{C})$, $62.4\left(\underline{\mathrm{CH}}_{2}\right), 60.3\left(2 \mathrm{O}_{\mathrm{CH}}\right), 39.7\left(2 \mathrm{~N}_{2} \mathrm{H}_{2}\right), 15.2\left(2 \underline{\mathrm{CH}}_{3}\right)$.

$1^{4} 5^{4}$-Diethyl-3(1,3)-benzena-2,4-dioxa-6,9-dithia-1,5(3,5) (5,3)-di-(1,2,4-triazola)cyclononaphane (12b)

${ }^{1} \mathrm{H} \mathrm{NMR}\left(\mathrm{CDCl}_{3}\right): \delta 7.25-6.64(\mathrm{~m}, 4 \mathrm{H}$, Aromatic $\mathrm{H}), 5.15$ $\left(\mathrm{s}, 4 \mathrm{H}, 2 \mathrm{OCH}_{2}\right), 4.02$ (q, 4H, 2NCH $), 3.10\left(\mathrm{t}, 4 \mathrm{H}, 2 \mathrm{SCH}_{2}\right)$, $1.26\left(\mathrm{t}, 6 \mathrm{H}, 2 \mathrm{CH}_{3}\right) \cdot{ }^{13} \mathrm{CNMR}\left(\mathrm{CDCl}_{3}\right): \delta 152.5,147.8(2 \mathrm{C}=\mathrm{N})$, 158.6, 112.4, 107.9, 102.1 (6 Aromatic C), $60.3\left(2 \mathrm{OCH}_{2}\right), 39.7$ $\left(2 \mathrm{NCH}_{2}\right), 37.2\left(2 \mathrm{SCH}_{2}\right), 15.2\left(2 \underline{\mathrm{CH}}_{3}\right)$.

$1^{4} 5^{4}$-Diethyl-3(1,3)-benzena-2,4-dioxa-6,10-dithia-1,5(3,5) (5,3)-di-(1,2,4-triazola)cyclodecaphane (12c)

${ }^{1} \mathrm{H}$ NMR $\left(\mathrm{CDCl}_{3}\right): \delta$ 7.25-6.64 $(\mathrm{m}, 4 \mathrm{H}$, Aromatic $\mathrm{H})$, $5.21\left(\mathrm{~s}, 4 \mathrm{H}, 2 \mathrm{OCH}_{2}\right), 4.10\left(\mathrm{q}, 4 \mathrm{H}, 2 \mathrm{NCH}_{2}\right), 3.62(\mathrm{t}, 4 \mathrm{H}$, $\left.2 \mathrm{SCH}_{2}\right), 3.02\left(\mathrm{p}, 2 \mathrm{H}, 1 \mathrm{CH}_{2}\right), 1.26\left(\mathrm{t}, 6 \mathrm{H}, 2 \mathrm{CH}_{3}\right) .{ }^{13} \mathrm{C} \mathrm{NMR}$ $\left(\mathrm{CDCl}_{3}\right): \delta 152.1,145.5(4 \underline{\mathrm{C}}=\mathrm{N}), 157.3,124.9,105.4$, 102.5 (6 Aromatic C), $55.4\left(2 \mathrm{O}^{\mathrm{CH}}{ }_{2}\right), 33.9\left(2 \mathrm{NCH}_{2}\right), 27.2$ $\left(2 \underline{\mathrm{S}}_{2}\right), 25.7\left(1 \underline{\mathrm{CH}}_{2}\right), 10.2\left(2 \underline{\mathrm{CH}}_{3}\right)$.

1454-Diethyl-3(1,3)-benzena-2,4-dioxa-6,11-dithia-1,5(3,5) (5,3)-di-(1,2,4-triazola)cycloundecaphane (12d)

${ }^{1} \mathrm{H} \mathrm{NMR}\left(\mathrm{CDCl}_{3}\right): \delta$ 7.25-6.64 $(\mathrm{m}, 4 \mathrm{H}$, Aromatic $\mathrm{H})$, $5.21\left(\mathrm{~s}, 4 \mathrm{H}, 2 \mathrm{OCH}_{2}\right), 4.10\left(\mathrm{q}, 4 \mathrm{H}, 2 \mathrm{NCH}_{2}\right), 3.01(\mathrm{t}, 4 \mathrm{H}$, $\left.2 \mathrm{SCH}_{2}\right), 1.72\left(\mathrm{p}, 4 \mathrm{H}, 2 \mathrm{CH}_{2}\right), 1.26\left(\mathrm{t}, 6 \mathrm{H}, 2 \mathrm{CH}_{3}\right) .{ }^{13} \mathrm{C} \mathrm{NMR}$ $\left(\mathrm{CDCl}_{3}\right): \delta 151.0,150.8(4 \underline{\mathrm{C}}=\mathrm{N}), 157.3,130.2,110.0$, 104.0 (6 Aromatic C), $60.6\left(2 \mathrm{O}^{-} H_{2}\right), 39.2\left(2 \mathrm{NCH}_{2}\right), 32.8$ $\left(2 \underline{\mathrm{CH}}_{2}\right), 27.8\left(2 \underline{\mathrm{CH}}_{2}\right), 15.2\left(2 \underline{\mathrm{CH}}_{3}\right)$. 
$1^{4} 5^{4}$-Diethyl-3(1,3)-benzena-2,4-dioxa-6,12-dithia-1,5(3,5) (5,3)-di-(1,2,4-triazola)cyclododecaphane (12e)

${ }^{1} \mathrm{H}$ NMR $\left(\mathrm{CDCl}_{3}\right): \delta$ 7.25-6.64 $(\mathrm{m}, 4 \mathrm{H}$, Aromatic $\mathrm{H})$, $5.20\left(\mathrm{~s}, 4 \mathrm{H}, 2 \mathrm{OCH}_{2}\right), 4.10\left(\mathrm{q}, 4 \mathrm{H}, 2 \mathrm{NCH}_{2}\right), 3.01(\mathrm{t}, 4 \mathrm{H}$, $\left.2 \mathrm{SCH}_{2}\right), 1.40-1.32\left(\mathrm{~m}, 6 \mathrm{H}, 3 \mathrm{CH}_{2}\right), 1.26\left(\mathrm{t}, 6 \mathrm{H}, 2 \mathrm{CH}_{3}\right) \cdot{ }^{13} \mathrm{C}$ $\operatorname{NMR}\left(\mathrm{CDCl}_{3}\right): \delta 151.1,150.7(4 \underline{\mathrm{C}}=\mathrm{N}), 157.3,130.2,108.8$, 103.5 (6 Aromatic C), $59.8\left(2 \mathrm{OCH}_{2}\right), 39.3\left(2 \mathrm{NCH}_{2}\right), 34.0$, $29.0\left(4 \underline{\mathrm{S}}_{2}\right), 26.3\left(\underline{\mathrm{CH}}_{2}\right), 15.4\left(2 \underline{\mathrm{CH}}_{3}\right)$.

$1^{4}, 5^{4}$-Diphenyl-3(1,3)8(1,2)dibenzena-2,4-dioxa-6,10dithia-1,3(3,5)(5,3)-di-(1,2,4-triazola)cycldecaphane (12f)

${ }^{1} \mathrm{H}$ NMR $\left(\mathrm{CDCl}_{3}\right): \delta 7.36-6.61(\mathrm{~m}, 8 \mathrm{H}$, Aromatic $\mathrm{H})$, 5.10 (s, 4H, 20CH $), 4.64$ (s, 4H, 2SCH $), 3.98$ (q, 4H, $\left.2 \mathrm{NCH}_{2}\right), 1.26\left(\mathrm{t}, 6 \mathrm{H}, 2 \mathrm{CH}_{3}\right) .{ }^{13} \mathrm{C} \mathrm{NMR}\left(\mathrm{CDCl}_{3}\right): \delta 151.1$, $147.8(4 \underline{C}=\mathrm{N}), 158.6,134.7-125.7,107.9,101.9(12$ Aromatic C), $60.4\left(2 \mathrm{O}^{-} H_{2}\right), 39.5\left(2 \mathrm{NCH}_{2}\right), 37.2\left(2 \underline{\mathrm{SCH}}_{2}\right)$, $15.1\left(2 \mathrm{CH}_{3}\right)$.

1,3-Di-[4-anilino-5(2'-hydroxyphenyl)-1,2,4-triazol-3-yl] mercapto propane $(\mathbf{1 5})$

${ }^{1} \mathrm{H}$ NMR $\left(\mathrm{CDCl}_{3}\right): \delta 11.12$ (s, 2H, 2OH, D, $\mathrm{O}$ exchangeable), 7.84-6.94 (m, 18H, Aromatic H), 5.72 (s, 2H, 2NH, D O exchangeable), 3.42 ( $\left.\mathrm{t}, 4 \mathrm{H}, 2 \mathrm{SCH}_{2}\right), 2.32$ (p, 2H, $\left.\mathrm{CH}_{2}\right) \cdot{ }^{13} \mathrm{C} \mathrm{NMR}\left(\mathrm{CDCl}_{3}\right): \delta 156.3(2 \mathrm{~S}-\underline{\mathrm{C}}=\mathrm{N}), 153.4(2 \mathrm{C}=\mathrm{N})$, 156.2-113.2 (24 Aromatic C), $30.4\left(2 \underline{\mathrm{C}}_{2}\right), 28.5\left(-\underline{\mathrm{CH}}_{2}\right)$.

$1^{4}, 9^{4}$-Dianilino-2,8(1,2)-dibenza-3,7-dioxa-10,14-dithia1(3,5)9(5,3)-di-(1,2,4-triazola)cyclotetradecaphane (16a)

${ }^{1} \mathrm{H}$ NMR $\left(\mathrm{CDCl}_{3}\right): \delta$ 7.68-6.74 $(\mathrm{m}, 18 \mathrm{H}$, Aromatic $\mathrm{H}), 5.87$ (s, 2H, 2NH), $4.18\left(\mathrm{t}, 4 \mathrm{H}, 2 \mathrm{OCH}_{2}\right), 3.62(\mathrm{t}, 4 \mathrm{H}$, $2 \mathrm{SCH}_{2}$ ), 2.41 (p, 2H, $\left.\mathrm{CH}_{2}\right), 2.23$ (p, 2H, $\left.\mathrm{CH}_{2}\right)$. MS (DI) $(\mathrm{m} / \mathrm{z}): 648\left(\mathrm{M}^{+}\right)$.

$1^{4}, 10^{4}$-Dianilino-2,9(1,2)-dibenza-3,8-dioxa-11,15-dithia1(3,5)10(5,3)-di-(1,2,4-triazola)cyclopentadecaphane (16b)

${ }^{1} \mathrm{H} \mathrm{NMR}\left(\mathrm{CDCl}_{3}\right): \delta 7.86-7.10(\mathrm{~m}, 18 \mathrm{H}$, Aromatic $\mathrm{H})$, $5.72(\mathrm{~s}, 2 \mathrm{H}, 2 \mathrm{NH}), 3.82\left(\mathrm{~s}, 4 \mathrm{H}, 2 \mathrm{OCH}_{2}\right), 3.42\left(\mathrm{t}, 4 \mathrm{H}, \mathrm{SCH}_{2}\right)$, $2.34\left(\mathrm{p}, 2 \mathrm{H}, \mathrm{CH}_{2}\right), 2.06\left(\mathrm{~m}, 4 \mathrm{H}, 2 \mathrm{CH}_{2}\right)$.

$1^{4}, 9^{4}$-Dianilino-3,7-dioxa-10,14-dithia-1(3,5)9(5,3)-di(1,2,4-triazola)-2,5,8(1,2)-tribenza cyclotetradecaphane (16c)

${ }^{1} \mathrm{H}$ NMR $\left(\mathrm{CDCl}_{3}\right): \delta$ 7.83-6.81 $(\mathrm{m}, 22 \mathrm{H}$, Aromatic $\mathrm{H}), 5.72(\mathrm{~s}, 2 \mathrm{H}, 2 \mathrm{NH}), 4.62\left(\mathrm{~s}, 4 \mathrm{H}, 2 \mathrm{OCH}_{2}\right), 3.42(\mathrm{t}, 4 \mathrm{H}$, $\left.2 \mathrm{SCH}_{2}\right), 2.32\left(\mathrm{p}, 2 \mathrm{H}, \mathrm{CH}_{2}\right)$.

\section{General procedure for the synthesis of $\mathbf{8} \boldsymbol{a}-\boldsymbol{b}$}

Isophthalic acid dihydrazide $(0.01 \mathrm{~mol})$ and phenyl or $o$-tolyl isothiocyanate $(0.02 \mathrm{~mol})$ were refluxed in methanol
$(50 \mathrm{~mL})$ for $4 \mathrm{~h}$. The compound formed was filtered, washed with water, was added to $10 \%$ aqueous alkali $(100 \mathrm{~mL})$ and heated on water bath for $4 \mathrm{~h}$. The reaction mixture was poured over crushed ice. This cold solution was then filtered to remove some trace particles. Obtained filtrate was neutralized with dilute aqueous hydrochloric acid to obtain a solid compound, which was filtered, washed with cold water and recrystallised from aqueous DMF.

1,3-Bis-(5-mercapto-4-phenyl-1,2,4-triazol-3-yl)benzene (8a)

${ }^{1} \mathrm{H}$ NMR (DMSO d $): \delta 14.31$ (s, 2H, 2NH), 7.66-7.41 (m, 14H, Aromatic H). ${ }^{13} \mathrm{C}$ NMR (DMSO d $): \delta 169.1$ $(2 \mathrm{C}=\mathrm{S}), 150.0(2 \mathrm{C}=\mathrm{N}), 134.5-126.6(18$ Aromatic C $) . \mathrm{MS}$ (DI) $(\mathrm{m} / \mathrm{z}): 428\left(\mathrm{M}^{+}\right)$.

1,3-Bis-[5-mercapto-4-(2'-methyl)phenyl-1,2,4-triazol3-yl]benzene $(8 \boldsymbol{b})$

${ }^{1} \mathrm{H}$ NMR (DMSO d $): \delta 14.18(\mathrm{~s}, 2 \mathrm{H}, 2 \mathrm{NH}), 7.39-7.18$ $(\mathrm{m}, 12 \mathrm{H}$, Aromatic $\mathrm{H}), 1.91\left(\mathrm{~s}, 6 \mathrm{H}, 2 \mathrm{CH}_{3}\right) .{ }^{13} \mathrm{C} \mathrm{NMR}$ $\left(\mathrm{DMSO}_{6}\right): \delta 168.2(2 \mathrm{C}=\mathrm{S}), 149.3(2 \mathrm{C}=\mathrm{N}), 17.2\left(2 \mathrm{CH}_{3}\right)$, 135.7-126.2 (18 Aromatic C). MS (DI) $(\mathrm{m} / \mathrm{z}): 456\left(\mathrm{M}^{+}\right)$.

Synthesis of 1,3-bis-[(4-ethyl-3-mercapto-1,2,4-triazol-3yl)methyleneoxy]benzene (11)

Benzene-1,3-dioxyacetic acid $\mathbf{1 0}$ (2.26 g, 0.01 mol) and 4-ethyl-3-thiosemicarbazide 2a (2.38 g, 0.02 mol) were thoroughly mixed using mortar and pestle. This mixture was then fused in oil-bath maintaining temperature $140{ }^{\circ} \mathrm{C}$ for $4 \mathrm{~h}$. The reaction mixture was then cooled and dumped into ice. The resulting brown colored solid filtered off, washed with 5\% sodium bicarbonate solution followed by water till $\mathrm{pH}$ becomes neutral and recrystallized from aqueous DMF, $3.13 \mathrm{~g}$ (85\%), mp $184{ }^{\circ} \mathrm{C} ;{ }^{1} \mathrm{H}$ NMR (DMSO d 6 ): $\delta 13.80$ (s, 2H, 2x-NH), 7.28-6.72 (m, 4H, Aromatic H), $5.23\left(\mathrm{~s}, 4 \mathrm{H}, 2 \mathrm{OCH}_{2}\right)$, $4.01\left(\mathrm{q}, 4 \mathrm{H}, 2 \mathrm{NCH}_{2}\right), 1.26\left(\mathrm{t}, 6 \mathrm{H}, 2 \mathrm{CH}_{3}\right)$.

General procedure for the synthesis of 4-anilino-5-(2'hydroxyphenyl)-3-mercapto-1,2,4-triazole (14)

Salicylic acid hydrazide 13 (1.52 g, $0.01 \mathrm{~mol})$ was dissolved in ethanol $(40 \mathrm{~mL})$ in the presence of potassium hydroxide $(1.12 \mathrm{~g}, 0.02 \mathrm{~mol})$ and cooled at $5{ }^{\circ} \mathrm{C}$. To this cold solution carbon disulphide $(1.14 \mathrm{~g}, 0.015 \mathrm{~mol})$ was added under stirring. The precipitated dithiocarbamate salt intermediate was filtered off, washed with petroleum ether and dried. This salt was fused with phenylhydrazine (1.08 g, $0.01 \mathrm{~mol})$ at $140{ }^{\circ} \mathrm{C}$ for $6 \mathrm{~h}$. The reaction mixture was then poured onto cold water and filtered to remove traces of inorganic material. The filtrate was neutralized with dilute 
aqueous hydrochloric acid till $\mathrm{pH}$ of the solution became neutral. The resulting pale yellow solid was filtered off, washed with cold water and recrystallized from aqueous DMF. ${ }^{1} \mathrm{H}$ NMR (DMSO d $)$ ): $\delta 13.84$ (s, 1H, S=C-NH, D 2 exchangeable), 10.28 (s, 1H, OH, $\mathrm{D}_{2} \mathrm{O}$ exchangeable), 7.43-6.84 (m, 9H, Aromatic H), $5.82\left(\mathrm{~s}, 1 \mathrm{H}, \mathrm{NH}, \mathrm{D}_{2} \mathrm{O}\right.$ exchangeable). ${ }^{13} \mathrm{C}$ NMR (DMSO d $): \delta 164.2(2 \mathrm{C}=\mathrm{S})$, 148.2 (2 C=N), 154.3-113.1 (12 Aromatic C).

\section{Acknowledgments}

The help rendered by the Regional Sophisticated Instrumentation Centres (RSIC), the Indian Institute of Technology (IIT) and the Tata Institute of Fundamental Research (TIFR); Department of Chemistry, University of Pune, and Department of Chemistry, Institute of Science, Mumbai, is gratefully acknowledged.

\section{Supplementary Information}

${ }^{1} \mathrm{H}$ NMR, ${ }^{13} \mathrm{C}$ NMR data is available free of charge at http://jbcs.sbq.org.br, as PDF file.

\section{References}

1. Graf, E.; Lehn, J. M.; Helv. Chim. Acta 1981, 64, 1040; Dietrich, B.; Hosseini, M. W.; Lehn, J. M.; Session, R. B.; Helv. Chim. Acta 1983, 66, 1262; Hosseini, M. W.; Lehn, J. M.; Mertes, M. P.; Helv. Chim. Acta 1983, 66, 2444; Kumar, A.; Mageswaran, S.; Sutherland, I. O.; Tetrahedron 1986, 42, 3291; Bradshaw, J. S.; Izatt, R. M.; Acc. Chem. Res. 1997, 30, 338.

2. Newkome, G. R.; Traynham, J. G.; Baker, G. R. In Comprehensive Heterocyclic Chemistry; Katritzky, A. R., ed.; Pergamon Press: London, 1984, vol. 5, p. 763; Stark, C. M.; Liotta, C.; Halpern, M.; Phase Transfer Catalysis, Fundamentals: Applications and Industrial Perspective, Chapman and Hall: New York, 1994.

3. Bradshaw, J. S.; Izzat, R. M.; Bordunov, A. V.; Zhu, C. Y.; Hathaway, J. K. In Comprehensive Supramolecular Chemistry; Gokel, G. W., ed.; Pergamon: New York, 1996, vol.1, p. 35.

4. Pedersen, C. J.; J. Am. Chem. Soc. 1970, 89, 2495.

5. Singh, H.; Yadav, L. D. S.; Bhattacharya, B. K.; J. Indian Chem. Soc. 1979, 56, 1013; Desai, N. C.; Indian J. Chem. 1993, 32B, 343; Feng, X. M.; Chen, R.; Liu, X. C.; Zhang, Z. Y.; Chin. J. Appl. Chem. 1991, 8, 28; Upadhyay, P. S.; Vansdadia, R. N.; Baxi, A. J.; Indian J. Chem. 1990, 29B, 793; Mohan, J.; Anjaneyula, G. S. R.; Sudhir, S.; Arora, D. R.; J. Indian Chem. Soc. 1989, 66, 330; Ghorab, M. M.; El-Sharief, A. M. Sh.; Ammar, Y. A.; Mohamed, Sh. I.; Farmaco 2000, 55, 354.

6. Zhang, Z. Y.; Chen, X. L.; Wei, L.; Ma, Z. L.; Chem. Res. Chin. Univ. 1991, 7, 129.
7. Naito, Y.; Akahoshi, F.; Takeda, S.; Okada, T.; Kajii, M.; Nishimura, H.; Sugiura, M.; Fukaya, C.; Kagitani, Y.; J. Med. Chem. 1996, 39, 3019.

8. Narang, A. S.; Vince, R.; J. Med. Chem. 1977, 20, 1684; De Clercq, E.; J. Clin. Virol. 2004, 30, 115.

9. Collin, X.; Sauleau, A.; Coulon, J.; Bioorg. Med. Chem. Lett. 2003, 13, 2601; Holla, B. S.; Kalluraya, B.; Indian J. Chem. 1988, 27B, 683; Giri, S.; Singh, H.; Yadav, L. D. S.; Khare, R. K.; J. Indian Chem. Soc. 1978, 55, 168.

10. Kidwai, M.; Sapra, P.; Misra, P.; Saxena, R. K.; Singh, M.; Bioorg. Med. Chem. 2001, 9, 217.

11. George, T.; Mehta, D. V.; Tahilramani, R.; David, J.; Talwalker, P. K.; J. Med. Chem. 1971, 14, 335; PapakonstantinouGaroufalias, S.; Pouli, N.; Marakos, P.; Chytyroglou-Ladas, A.; Farmaco 2002, 57, 973; Sengupta, A. K.; Misra, H. K.; J. Indian Chem. Soc. 1981, 58, 508.

12. Ghorab, M. M.; Abdel- Hamide, S. G.; El-Gaby, M. S. A.; ElSayed, S. M.; Acta Pharm. 1999, 49, 1.

13. Andotra, C. S.; Sharma, S. K.; Proc. Natl. Acad. Sci. India 1988 , $58 A, 215$.

14. Hester, J. B.; Rudzik, A. D.; Kamdar, B. V.; J. Med. Chem. 1971, $14,1078$.

15. Milton, N. G. N.; Neurotoxicology 2001, 22, 767.

16. Burell, G.; Evans, J. M.; Hadley, M. S.; Hicks, F.; Stemp, G.; Bioorg. Med. Chem. Lett. 1994, 4, 1285; Ghorab, M. M.; Abdel- Hamide, S. G.; Ali, G. M.; El-Sayed, H. S.; Shaurub, H.; Pestic. Sci. 1996, 48, 31; Mody, M. K.; Prasad, A. R.; Ramalingham, T.; Suttur, P. B.; J. Indian Chem. Soc. 1982, 59, 769.

17. Wadsworth, J. H.; Jenkins, S. M.; Orlek, B. S.; Cassidy, F.; Clark, M. S. G.; Brown, F.; Riley, G. J.; Graves, D.; Hawkins, J.; Naylor, C. B.; J. Med. Chem. 1992, 35, 1280; Jenkins, S. M.; Wadsworth, H.; Bromidge, S.; Orlek, B. S.; Wyman, P. A.; Riley, G. J.; Hawkins, J.; J. Med. Chem. 1992, 35, 2392; Chen, C.; Dagnino, R.; Huang, C. Q.; McCarthy, J. R.; Grigoriadis, D. E.; Bioorg. Med. Chem. Lett. 2001, 11, 3165; Neumann-Schultz, B.; Unger, L.; Blumbach, K.; Starck, D.; Schoebel, D.; Treiber, H.; WO 00420382000.

18. Thompson, S. K.; Eppley, A. M.; Frazee, J. S.; Darcy, M. G.; Lum, R. T.; Tomaszeck, T. A.; Ivanoff, L. A.; Morris, J. F.; Sternberg, E. J.; Lambert, D. M.; Fernandez, A. V.; Patteway, S. R.; Meek, T. D.; Metcalf, B. W.; Gleason, J. G.; Bioorg. Med. Chem. Lett. 1994, 4, 2441.

19. Duncia, J. V.; Santella, J. B.; Higley, C. A.; VanAtten, M. K.; Weber, P. C.; Alexander, R. S.; Kettner, C. A.; Pruitt, J. R.; Liauw, A. Y.; Quan, M. L.; Knabb, R. M.; Wexler, R. R.; Bioorg. Med. Chem. Lett. 1998, 8, 775.

20. Potts, K. T.; Chem. Rev. 1961, 61, 87.

21. Chande, M. S.; Godbole, A. A.; Sajithkumar, C.; Heteroat. Chem. 2003, 14, 273; Chande, M. S.; Godbole, A. A.; Coutinho, E.; Desai, P.; Ind. J. Chem. 2002, 42B, 397. 
22. Chande, M. S.; Athalye, S. S.; Synth. Commun. 1999, 29, 1711; Chande, M. S.; Athalye, S. S.; Synth. Commun. 2000, 30, 1667; Chande, M. S.; Athalye, S. S.; Godbole, A. A.; Indian J. Chem. 2004, 43B, 670; Chande, M. S.; Uchil, M. H.; Barve, P.A.; Heteroatom Chem. 2006, 14, 329; Chande, M. S.; Barve, P. A.; Khanwelkar, R. R.; Athalye, S. S.; Venkatraman, D.; Can. J. Chem. 2007, 85, 21.
23. Gokel, G. W.; Dishang, D. M.; Dimond, C. J.; J. Chem. Soc., Chem. Commun. 1980, 1053.

24. Xu, P.-F.; Sun, X.-W.; Zhang, L.-M.; Zhang, Z.-Y.; J. Chem. Res. (s) 1999, 2, 170.

25. Nomenclature as per the following: Powell, W. H.; Pure Appl. Chem. 1998, 70, 1513; Favre, H. A.; Hellwinkel, D.; Powel, W. H.; Smith, H. A.; Pure Appl. Chem. 2002, 74, 809.

Received: January 24, 2007 Published on the web: January 22, 2008 


\section{Chemoselective Synthesis of Novel Thiatriazolophanes}

\section{Madhukar S. Chande,* Kiran A. Puthamane, Pravin A. Barve, Rahul R. Khanwelkar and} Deepak S. Venkataraman

Department of Chemistry, The Institute of Science, 15, Madam Cama Road, Mumbai 400 032, India $1^{4}, 6^{4}$-Diethyl-7,12-dithia-1,6(3,5)-di-(1,2,4-triazola)cyclododecaphane, 5d.<smiles>CCCCCSc1nnc(SCCC)n1CC</smiles>

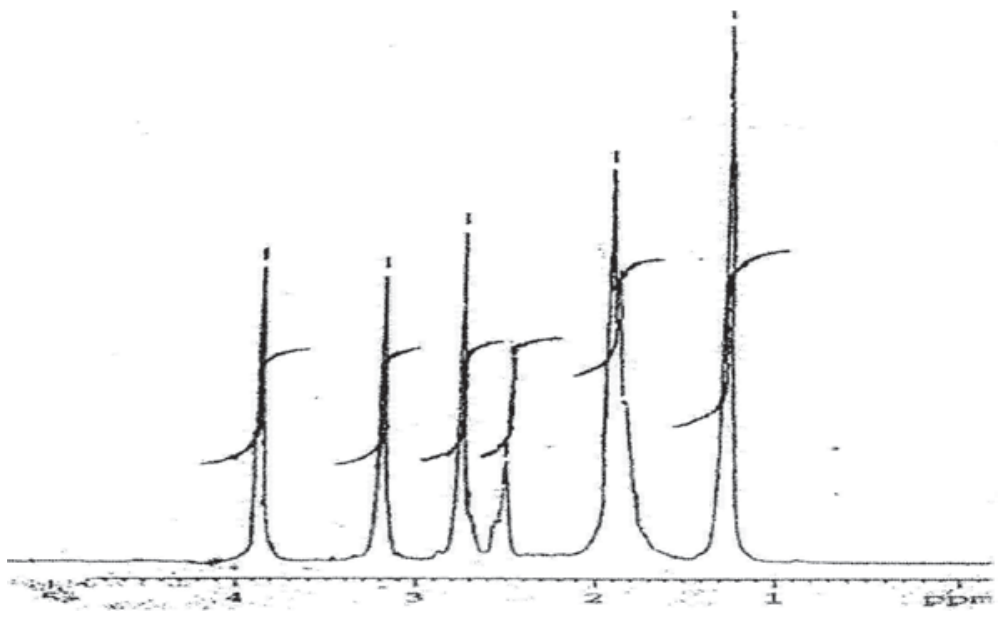

Figure S1. ${ }^{1} \mathrm{H}$ NMR for compound $\mathbf{5 d}$.

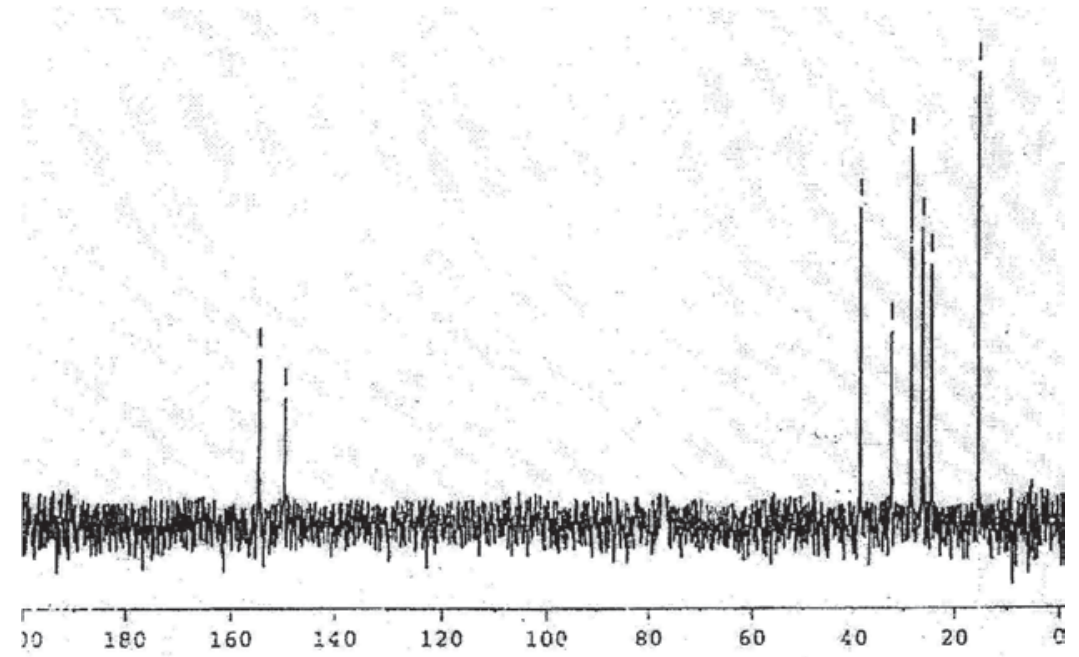

Figure S2. ${ }^{13} \mathrm{C}$ NMR for compound $\mathbf{5 d}$.

*e-mail: mschande@yahoo.com 
$1^{4}, 6^{4}$-Diphenyl-7,9-dithia-1,6(3,5)-di-(1,2,4-triazola)cyclononaphane $5 \mathbf{j}$.
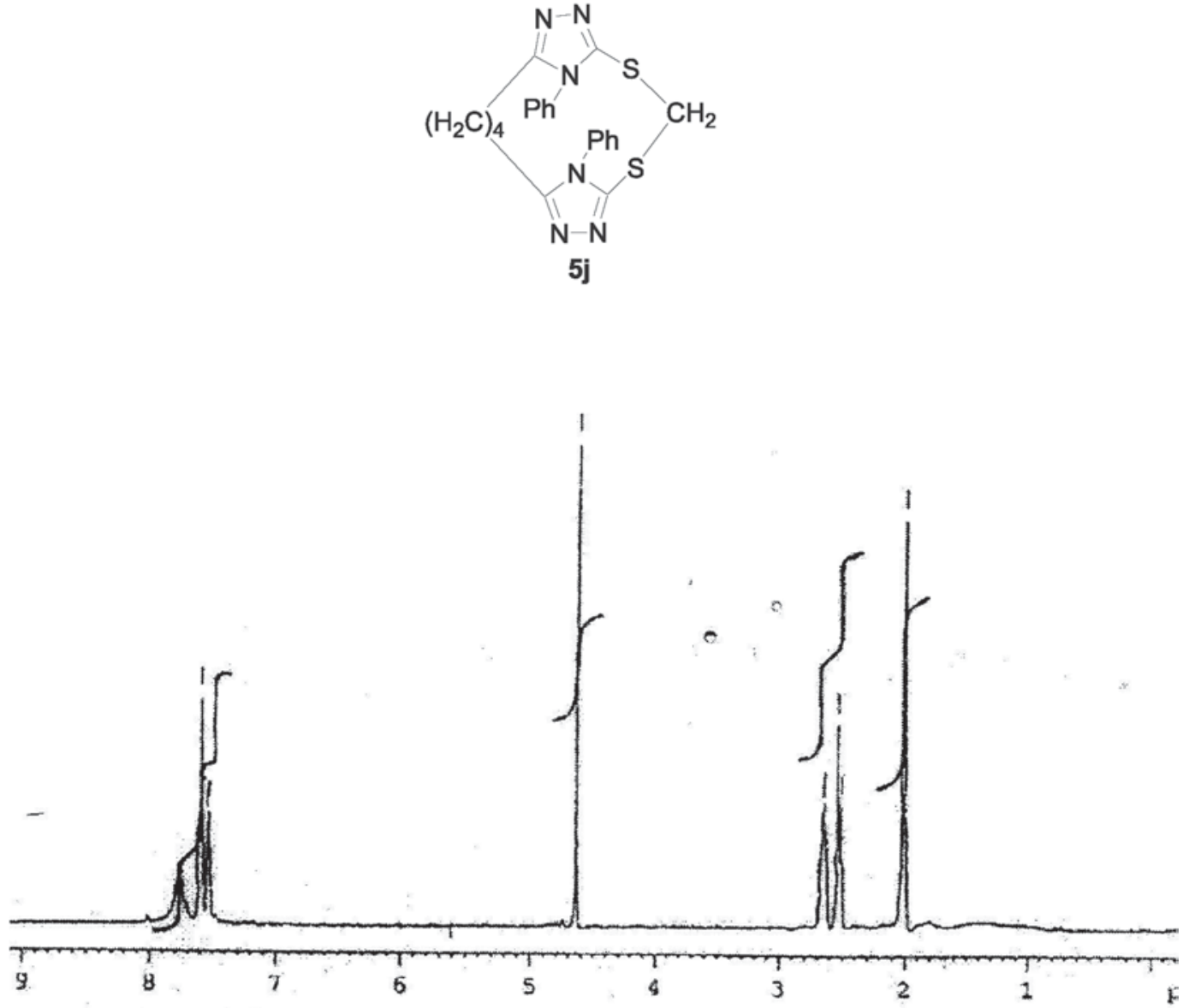

Figure S3. ${ }^{1} \mathrm{H}$ NMR for compound $\mathbf{5 j}$.

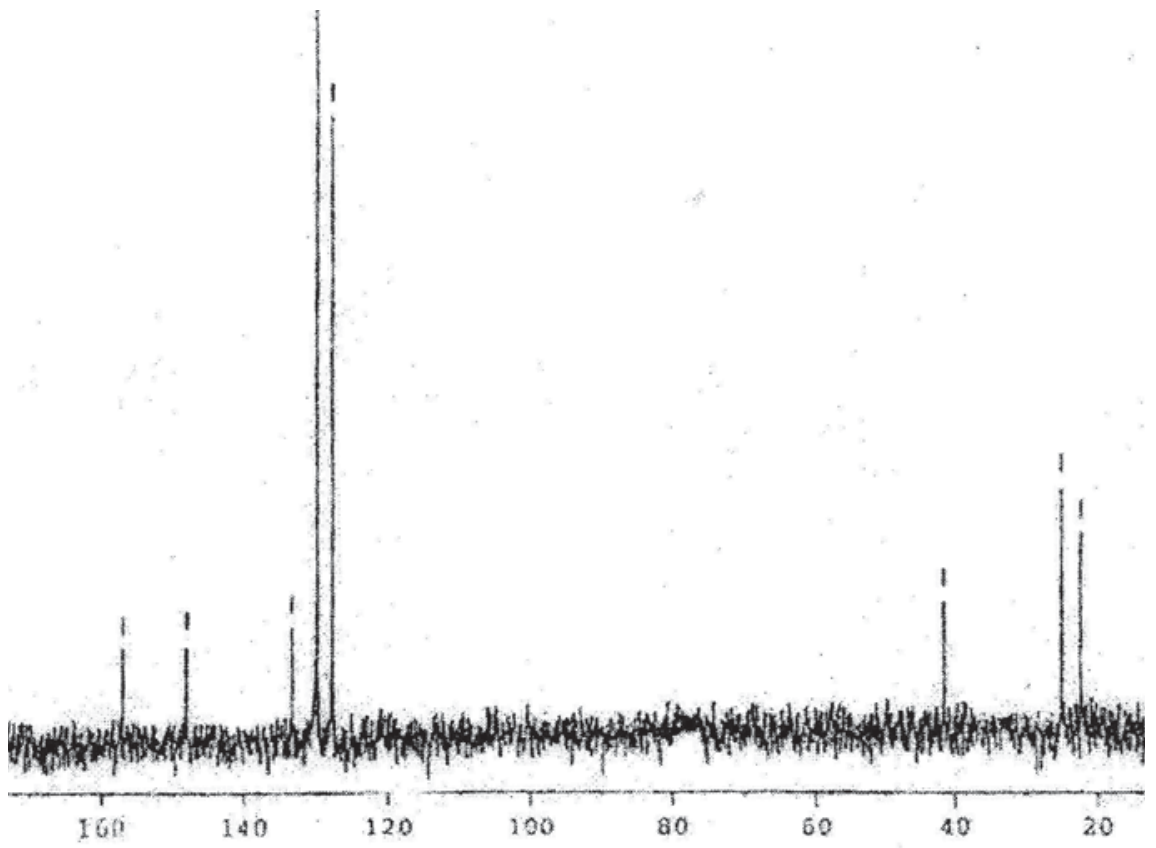

Figure S4. ${ }^{13} \mathrm{C}$ NMR for compound $\mathbf{5 j}$. 
$1^{4}, 6^{4}$-Diallyl-9(1,2)-benzena-7,11-dithia-1,3(3,5)(5,3)-di-(1,2,4triazola)cycloundecaphane $\mathbf{5 m}$.

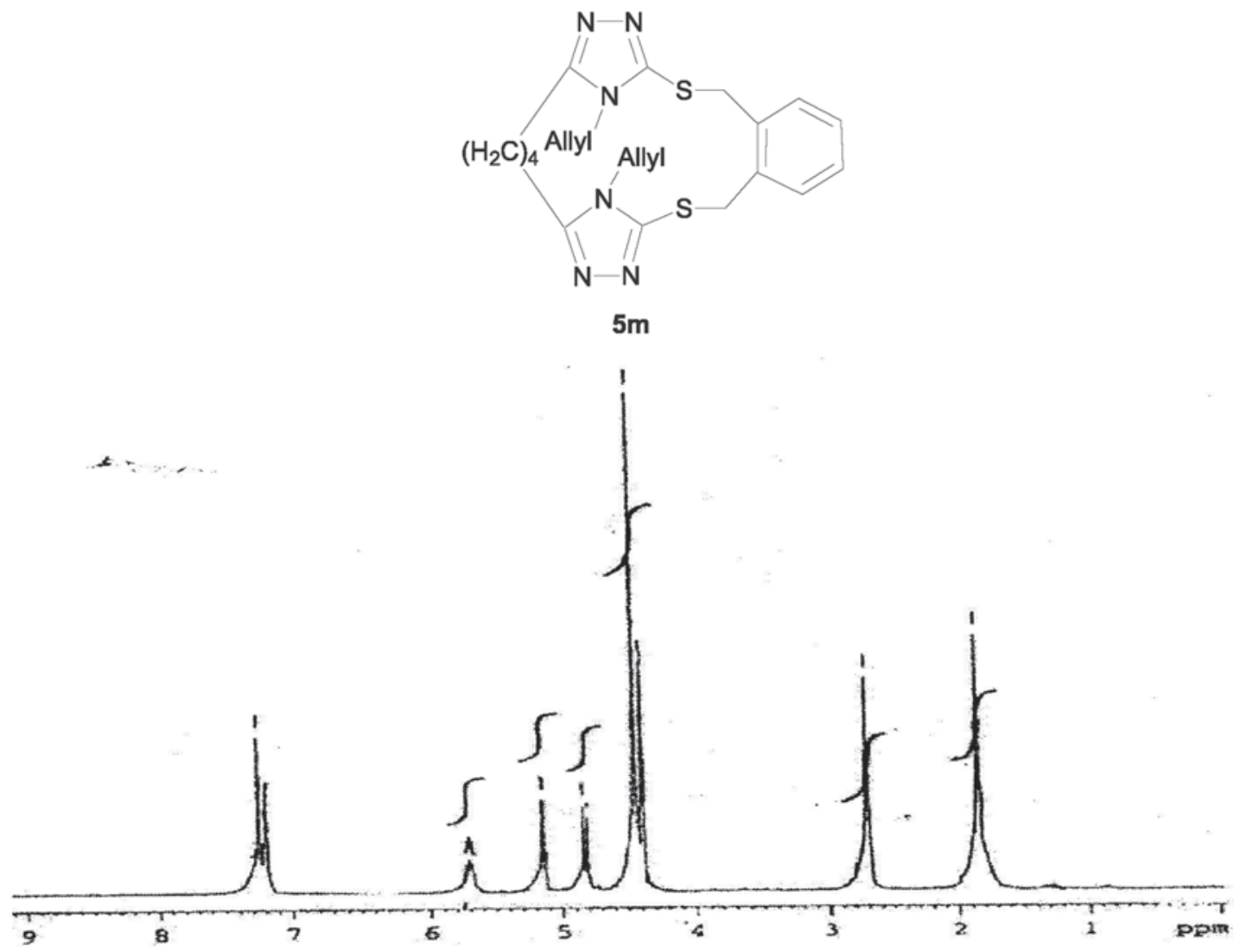

Figure S5. ${ }^{1} \mathrm{H}$ NMR for compound $\mathbf{5 m}$.

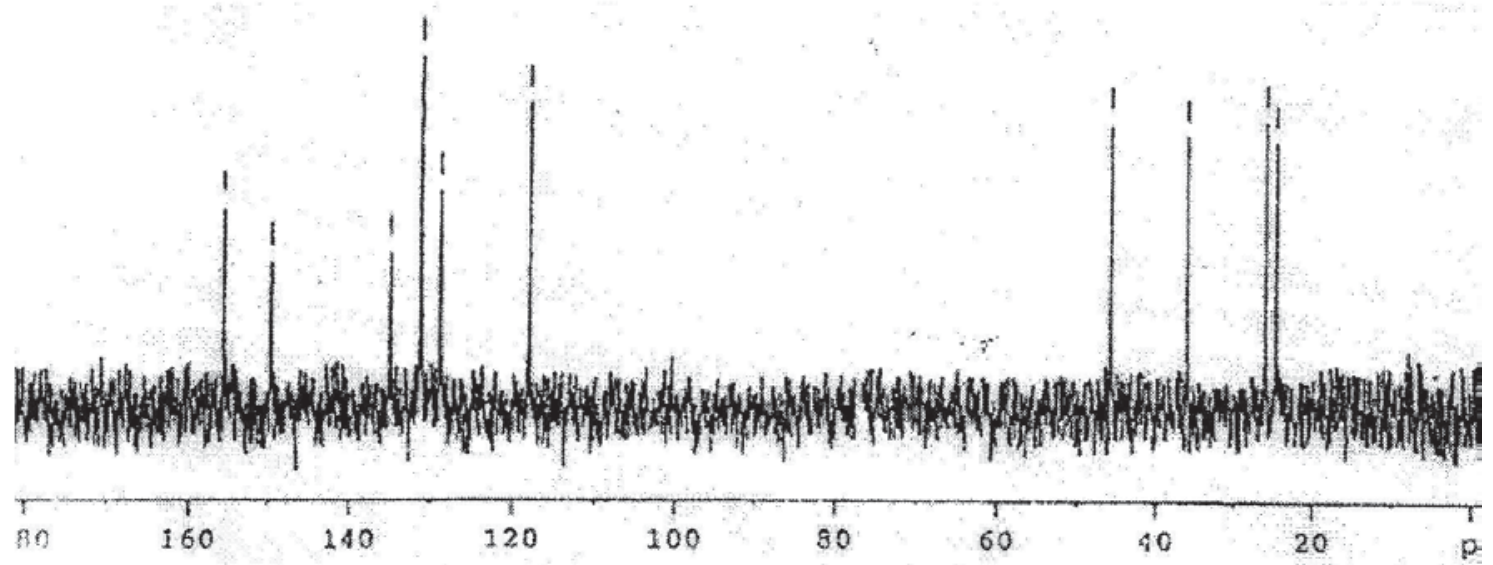

Figure S6. ${ }^{13} \mathrm{C}$ NMR for compound $\mathbf{5 m}$. 


\section{$1^{4}, 3^{4}$-Diphenyl-2(1,3)6(1,2)-dibenzena-4,8-dithia-1,3(3,5)(5,3)-di-(1,2,4 triazola)cycloctaphane $\mathbf{9 i}$.}
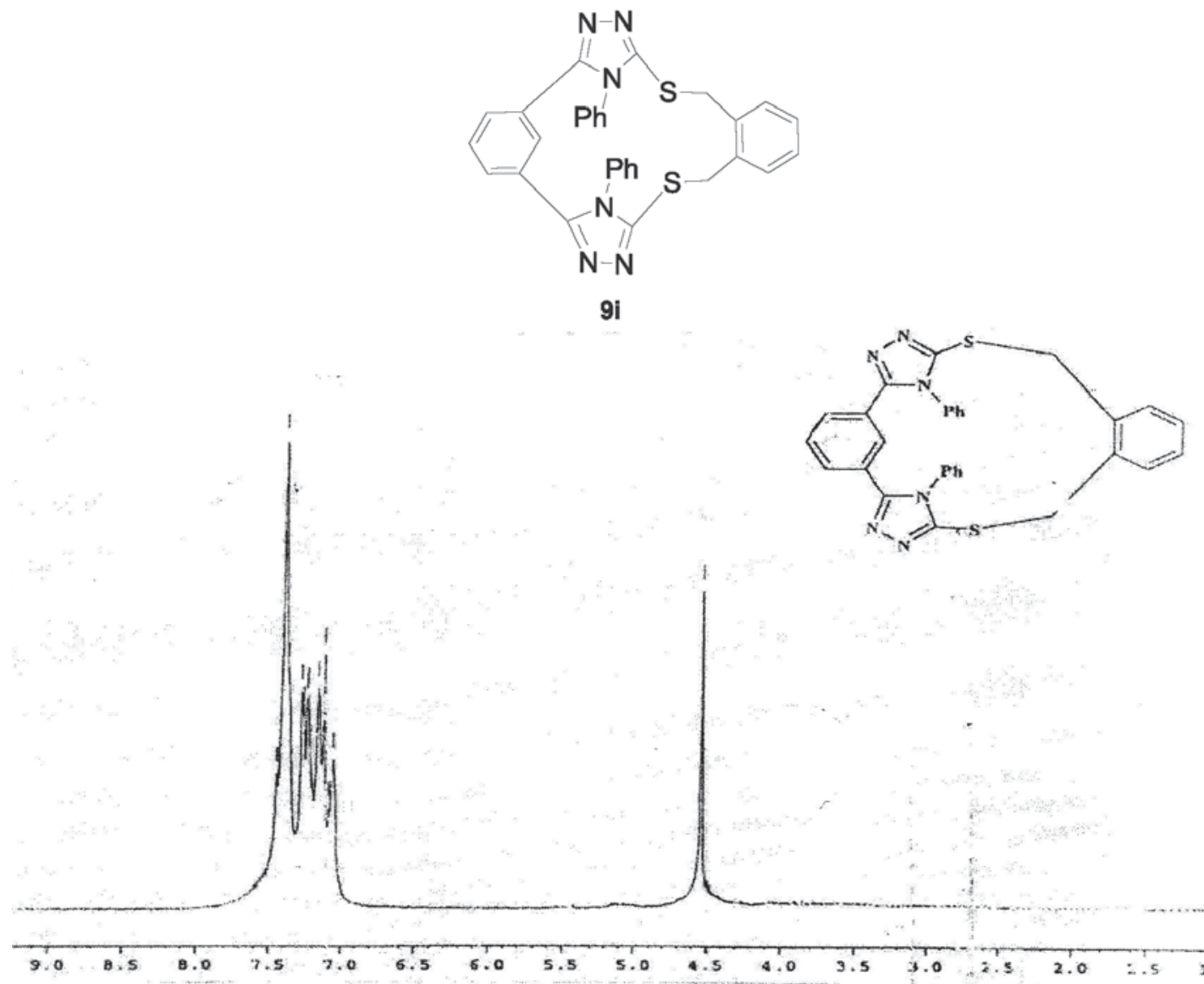

Figure S7. ${ }^{1} \mathrm{H}$ NMR for compound 9 i.

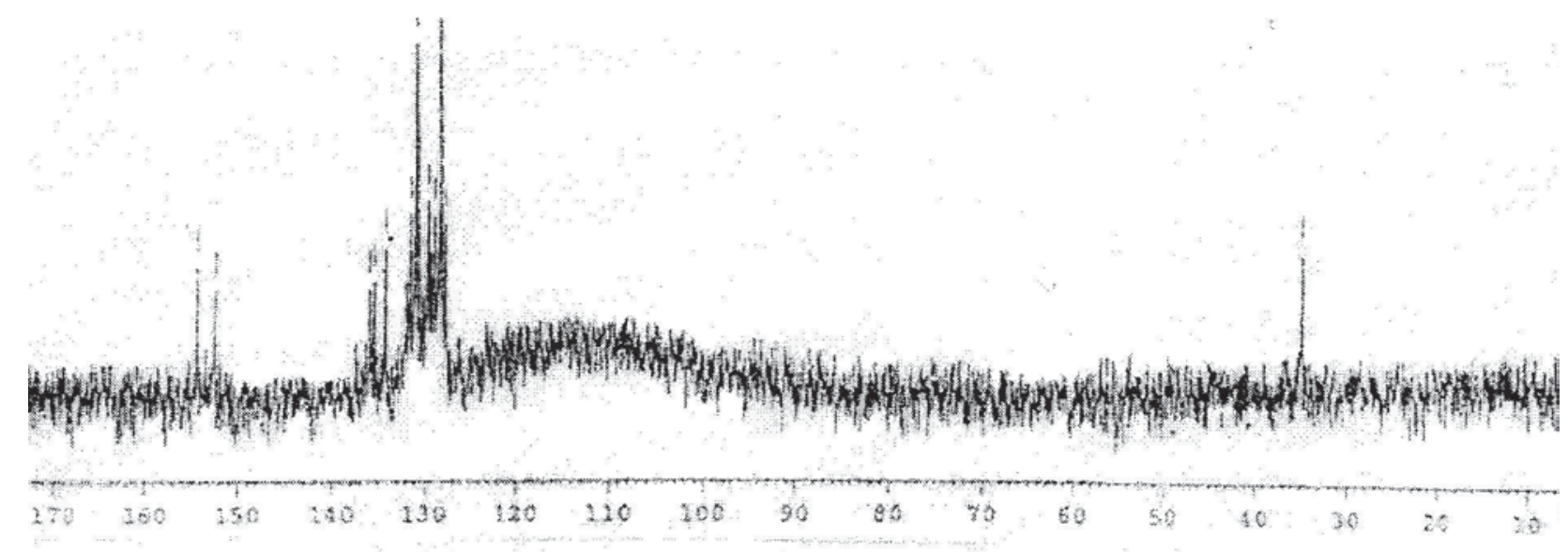

Figure S8. ${ }^{13} \mathrm{C}$ NMR for compound 9 i. 
$1^{4} 5^{4}$-Diethyl-3(1,3)-benzena-2,4-dioxa-6,10-dithia-1,5(3,5)(5,3)-di-(1,2,4triazola)cyclodecaphane 12c.

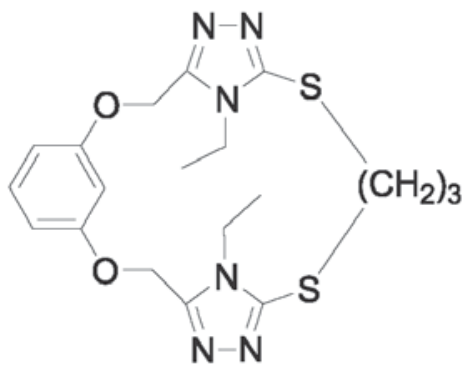

$12 c$

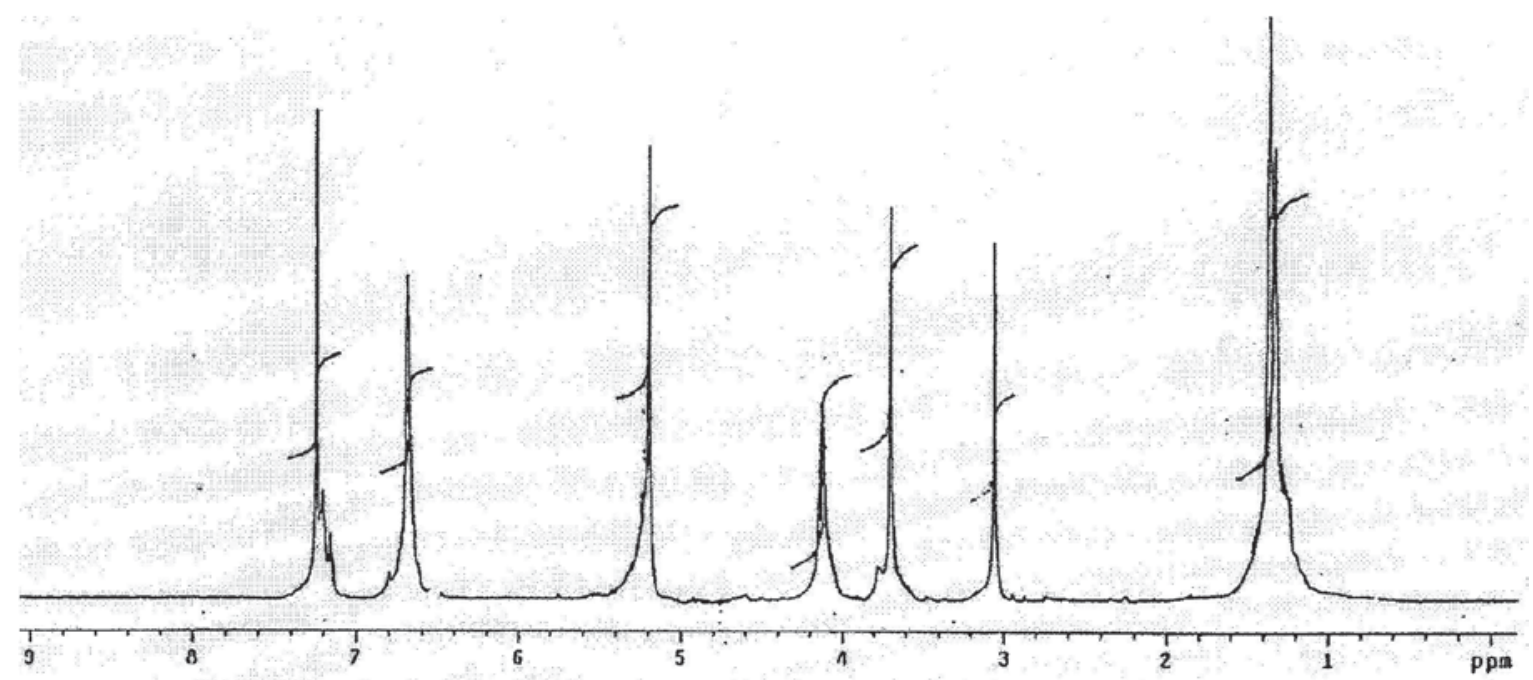

Figure S9. ${ }^{1} \mathrm{H}$ NMR for compound 12c.

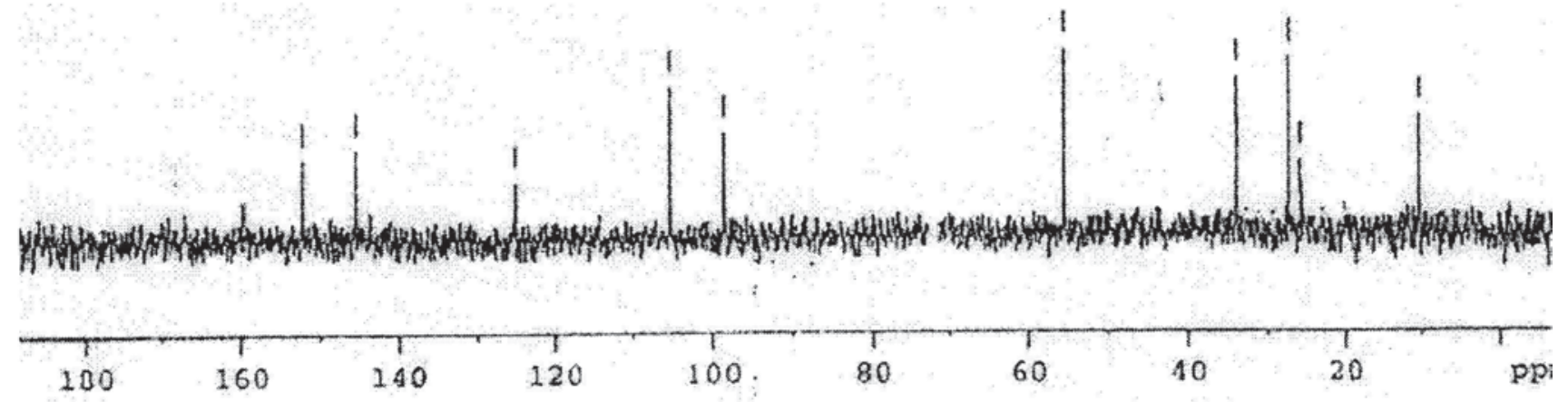

Figure S10. ${ }^{13} \mathrm{C}$ NMR for compound $\mathbf{1 2 c}$. 
$1^{4}, 9^{4}$-Dianilino-2,8(1,2)-dibenza-3,7-dioxa-10,14-dithia-1(3,5)9(5,3)-di-

(1,2,4-triazola)cyclotetradecaphane 16a.

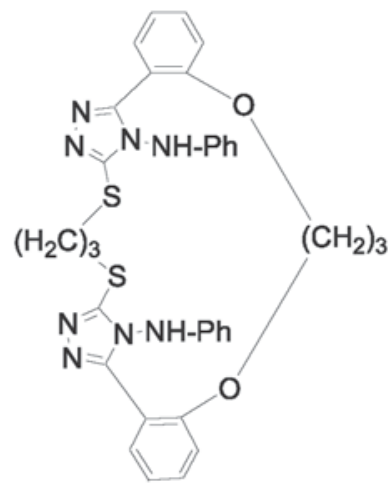

$16 a$

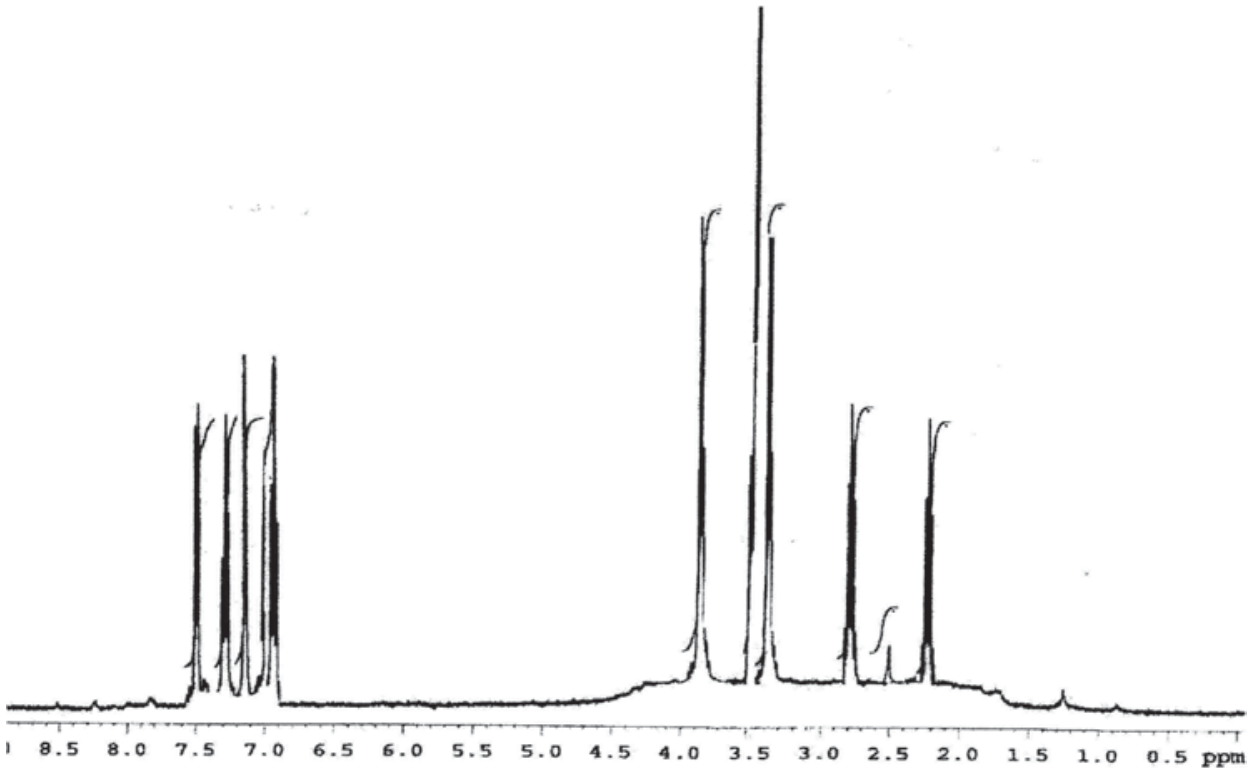

Figure S11. ${ }^{1} \mathrm{H}$ NMR for compound 16a.
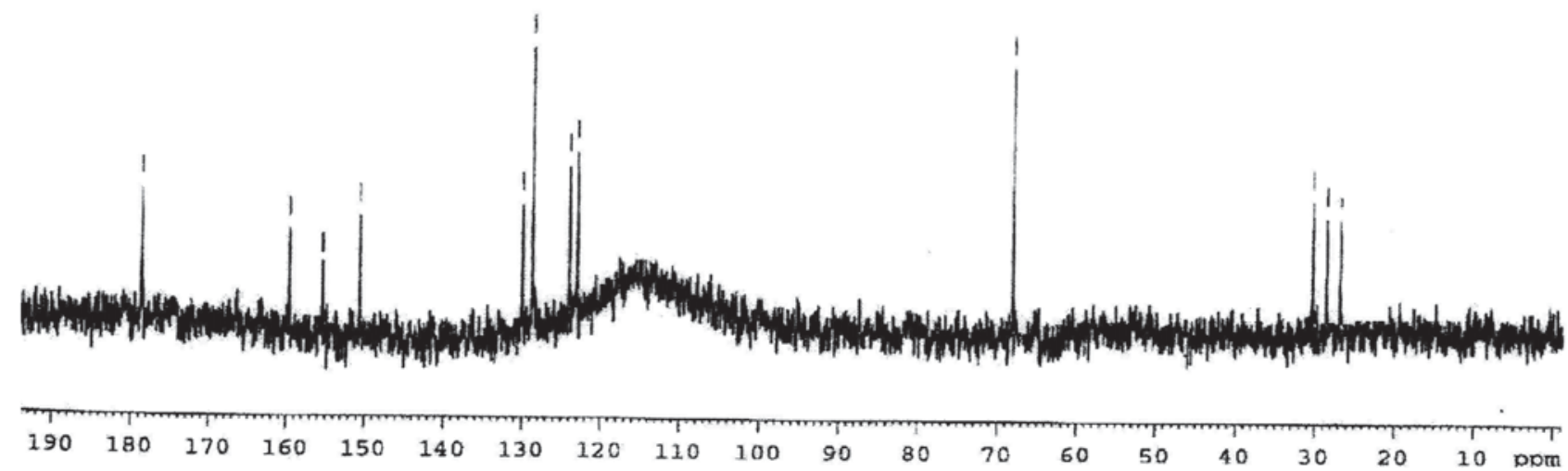

Figure S12. ${ }^{13} \mathrm{C}$ NMR for compound 16a. 